\title{
Multiline CO observations of MBM 32^
}

\author{
J.G.A. Wouterloot ${ }^{1,2}$, A. Heithausen ${ }^{1}$, W. Schreiber ${ }^{2}$, and G. Winnewisser ${ }^{2}$ \\ 1 Radioastronomisches Institut, Universität Bonn, Auf dem Hügel 71, D-53121 Bonn, Germany \\ 2 I. Physikalisches Institut, Universität zu Köln, Zülpicher Strasse 77, D-50937 Köln, Germany
}

Received October 5, 1999; accepted February 2, 2000

\begin{abstract}
We present a detailed study of the high latitude cloud MBM 32. Observations were made in the $J=(1-0)$, $(2-1)$, and $(3-2)$ transitions of ${ }^{12} \mathrm{CO}$ and in ${ }^{13} \mathrm{CO}(1-0)$ and $(2-1)$. These data were complemented by $21 \mathrm{~cm} \mathrm{HI}$ data and by IRAS 60 and $100 \mu \mathrm{m}$ data.

Our data show that MBM 32 consists of a main cloud component at $V_{\mathrm{lsr}}>2 \mathrm{~km} \mathrm{~s}^{-1}$ (mass about $\left.16.9 M_{\odot}\right)$, and a smaller component at $V_{\mathrm{lsr}}<0 \mathrm{~km} \mathrm{~s}^{-1}\left(4.1 M_{\odot}\right)$, in addition to some emission in between those two velocities $\left(<0.5 M_{\odot}\right)$.

We study the gas distribution and the CO line ratios within MBM 32, the latter also along a cut through both cloud components. We find that the ratios of different rotational transitions are constant within each cloud part. Only the ratio ${ }^{12} \mathrm{CO}(1-0) /{ }^{13} \mathrm{CO}(1-0)$ is lower in the cloud center. This suggests equal excitation conditions through MBM32 and line temperatures determined through clump filling factors.

All CO components show associated $\mathrm{HI}$ emission, but small velocity differences of typically $1 \mathrm{~km} \mathrm{~s}^{-1}$ exist between the $\mathrm{HI}$ and $\mathrm{CO}$ gas. The mass of associated HI gas is similar to the molecular mass for all components.

There is a good correlation between FIR, CO, and HI emission. The dust mass is about $0.073 M_{\odot}$, and the ratio of gas and dust mass (280) is lower than found from similar (IRAS) data for denser clouds, suggesting that the amount of dust colder than $20 \mathrm{~K}$ is relatively small.

We subdivide the $\mathrm{CO}$ data cubes in Gaussian shaped clumps; $40-50 \%$ of the CO emission can be assigned to the larger clumps. The remaining emission comes from either more extended clumps or from overlapping unresolved clumps.
\end{abstract}

Key words: ISM: clouds - ISM: individual objects: MBM 32 - ISM: molecules - radio lines: ISM

Send offprint requests to: J.G.A. Wouterloot, e-mail: wouterloot@astro.uni-bonn.de

* Tables 3 to 6 are only available in electronic form at the CDS via anonymous ftp to cdsarc.u-strasbg.fr (130.79.128.5) or via http://cdsweb.u-strasbg.fr/Abstract.html

\section{Introduction}

Molecular clouds are commonly thought to be the birth place of stars. The exact link between the mass of a star and the structure of the parent molecular cloud is not fully understood yet. It is therefore necessary to study molecular clouds in different galactic environments and with different degrees of gravitational binding to figure out possible differences which influence the formation of stars.

High latitude molecular clouds (HLCs) or galactic cirrus clouds are a class of molecular clouds where the conditions are not well suited to form stars. Their kinematics is largely dominated by turbulence and self-gravity plays only a minor role in their dynamics (Magnani et al. 1985; de Vries et al. 1987; Heithausen 1996). Detailed structural analysis of these clouds in comparison to those where the conditions for star-formation are more favourable will shed light on the star-forming process. Two further conditions make HLCs ideal targets for such a study: i) they are close to the Sun, and thus can be observed at high linear resolution even with small telescopes and ii) at their location at high galactic latitudes there is less confusion with fore- or background gas than there is close to the galactic plane.

In this paper we present a detailed study of MBM 32 (Magnani et al. 1985) which is located at $l=147^{\circ}, b=40^{\circ}$ in the complex of cirrus clouds known as the Ursa Major cirrus clouds (de Vries et al. 1987). MBM 32 harbours a dense core first detected in formaldehyde (Heithausen et al. 1987) and ammonia (Mebold et al. 1987). Based on a multi-transition $\mathrm{CO}$ and $\mathrm{NH}_{3}$ study, Schreiber et al. (1993) derived a kinetic temperature of $24_{-5}^{+10} \mathrm{~K}$ and were able to model 7 different $\mathrm{CO}$ transitions with a beam averaged $\left(4^{\prime}\right) \mathrm{H}_{2}$ column density of about $210^{20} \mathrm{~cm}^{-2}$ and a power law density distribution.

Over the last years we have continued to map MBM 32 in several $\mathrm{CO}$ transitions. These observations are presented in Sects. 2 and 3. Possible variations of the line ratios will help reveal the structure and properties of this cloud. To trace the atomic gas of this cloud we have also obtained a complete map in the $21 \mathrm{~cm} \mathrm{HI}$ line at 9!2 
resolution. In conjunction with corresponding IRAS maps we analyse the distibutions of atomic and molecular gas (Sect. 4). A distance towards MBM 32 (from e.g. star counts) has not yet been determined. Throughout this paper we shall assume a distance to MBM 32 of 100 pc.

\section{Observations}

\subsection{KOSMA observations}

Between 1989 and 1993 we have used the old (before the 1996 refurbishing) KOSMA 3-m telescope at Gornergrat (Switzerland) to observe ${ }^{12} \mathrm{CO}(J=1-0, J=2-1$ and $J=3-2)$ and ${ }^{13} \mathrm{CO}(J=1-0$, and $J=2-1)$ towards MBM 32. Based on a quick ${ }^{12} \mathrm{CO}(1-0)$ map consisting of 555 spectra on a $4^{\prime}$ raster made with a relatively low velocity resolution $\left(0.93 \mathrm{~km} \mathrm{~s}^{-1}\right)$, we obtained a higher velocity resolution map $\left(0.15 \mathrm{~km} \mathrm{~s}^{-1}\right)$ on a $2^{\prime}$ raster of a slightly smaller region. Subsequently we observed parts of the latter region on $2^{\prime}$ or $1^{\prime}$ rasters in the ${ }^{12} \mathrm{CO}(2-1$, and $3-2)$, and ${ }^{13} \mathrm{CO}(1-0$, and $2-1)$ transitions. One position (at offset $\left.\left(8^{\prime}, 19^{\prime}\right)\right)$ was also observed in $\mathrm{C}^{18} \mathrm{O}(2-1)$ and ${ }^{13} \mathrm{CO}(3-2)$ (see Schreiber et al. 1993). The observational parameters are summarized in Table 1 . In addition to the maps we made in ${ }^{12} \mathrm{CO}(2-1),{ }^{13} \mathrm{CO}(2-1)$, and in ${ }^{12} \mathrm{CO}(3-2)$, deeper observations in there transitions (typical rms noise levels $\left(T_{\mathrm{R}}^{*}\right)$ of $0.08,0.03$, and $0.10 \mathrm{~K}$ ) were made along a line through MBM 32 in order to study the line ratios. The raster size was in this case $1^{\prime}$ for both $(2-1)$ transitions and $40^{\prime \prime}$ for ${ }^{12} \mathrm{CO}(3-2)$. All offsets in this paper are given with respect to the MBM position $\alpha(1950)=9^{\mathrm{h}} 28^{\mathrm{m}} 42^{\mathrm{s}}, \delta(1950)=+66^{\circ} 5^{\prime}$. During all observations we frequently observed a calibration position towards offset $\left(4^{\prime}, 4^{\prime}\right)$ and found that intensities typically have uncertainties of about $10 \%$. The telescope and receivers used have been described by Winnewisser et al. (1986, 1990). The pointing accuracy was usually $10-15^{\prime \prime}$, but during daytime observations pointing errors might have been $30-40^{\prime \prime}$ due to thermal movements of the building. At 115 and $110 \mathrm{GHz}$ we used frequency switching and at other frequencies we observed in position switching mode using an off-source position $30^{\prime}$ south of MBM 32 . At 115 and $110 \mathrm{GHz}$ the atmospheric transmission was estimated by means of skydips. At higher frequencies the sky transmission was estimated by measuring the radiation temperature of blank sky at the elevation of MBM 32. Analogous to the standard chopper wheel method, we then corrected the intensities to the $T_{\mathrm{A}}^{*}$ scale. The backend consisted of a Medium Resolution (channel width $167 \mathrm{kHz}$; MRS), or a High Resolution (channel width $38.6 \mathrm{kHz}$; HRS) acousto-optical Spectrometer.

All intensities presented in this paper are on the scale $T_{\mathrm{R}}^{*}=T_{\mathrm{A}}^{*} / \eta^{\prime}$. The sizes of clumps in MBM 32 are several arcminutes, but the whole cloud has an extent of 0.5 to $1^{\circ}$. Therefore, to compare the intensities in the different transitions we corrected them by using the average value $\eta^{\prime}$ of the telescope efficiencies derived from Jupiter $\left(\eta_{\mathrm{mb}}\right)$ and from Moon measurements $\left(\eta_{\mathrm{fss}}\right)$. At $345 \mathrm{GHz}$ the efficiencies used differ for the three observing winters. The Jupiter measurements were in all cases made in good weather, but it is not known whether the efficiencies were constant during each winter or not. The values which we used are listed in Table 1. For the 115 and $110 \mathrm{GHz}$ measurements we applied in addition a correction $\eta_{\mathrm{R}} \eta_{\mathrm{rss}}=0.91$, which is already corrected for in the calibration at the higher frequencies. After applying these corrections we found a ratio $\int T_{\mathrm{R}}^{*}\left({ }^{12} \mathrm{CO}(1-0)\right) \mathrm{HRS} / \mathrm{MRS}$ of 1.25 (which we cannot explain), and to be able to use both data sets (and since the ${ }^{13} \mathrm{CO}$ data used for LTE column densities were also made with the HRS), we corrected the MRS data with this additional factor.

\subsection{Effelsberg HI observations}

We have used the Effelsberg 100-m telescope to obtain a fully-sampled $21 \mathrm{~cm} \mathrm{Hi} \mathrm{map} \mathrm{of} \mathrm{MBM} \mathrm{32.} \mathrm{The} \mathrm{beam}$ size at $1420 \mathrm{MHz}$ is 9.2 and we observed the cloud at the same $4^{\prime}$ grid as in the ${ }^{12} \mathrm{CO}(1-0)$ MRS map. The observations were made in frequency switching mode. The velocity resolution of $0.32 \mathrm{~km} \mathrm{~s}^{-1}$ was obtained with a 1024 channel autocorrelator. The rms noise in the spectra is $0.25-0.40 \mathrm{~K}$, with the lower value for positions which could be observed twice. The spectra were corrected for stray radiation (Kalberla et al. 1982) and calibrated using the standard position S7 (Williams 1973).

\section{Results}

\section{1. $C O$}

In Fig. 1a we show the distribution of the velocity integrated emission $\int T_{\mathrm{R}}^{*} \mathrm{~d} v$ of ${ }^{12} \mathrm{CO}(1-0)$ in $\mathrm{MBM} 32$, obtained from Gaussian fits to the lines. We used the high velocity resolution (HRS) $2^{\prime}$ raster data where possible and at the other positions we used the lower velocity resolution (MRS) $4^{\prime}$ data (put to the HRS intensity scale - see Sect. 2.1). We separately show the emission in three velocity intervals, separated by the LSR velocities from the Gaussian fits. The distribution of peak $T_{\mathrm{R}}^{*}$ is essentially the same as that of $\int T_{\mathrm{R}}^{*} \mathrm{~d} v$ (although details differ), i.e. line widths do not vary much within the region. The HI spectra east of $\alpha$-offset $-12^{\prime}$ clearly show three velocity components (see Sect. 3.2). Therefore, we also separated the $\mathrm{CO}$ emission in three velocity intervals, the main component of which is at velocities $V_{\mathrm{lsr}}$ between $2-5 \mathrm{~km} \mathrm{~s}^{-1}$ (the maximum $T_{\mathrm{R}}^{*}$ is $4.8 \mathrm{~K}$ at $\left(0^{\prime}, 4^{\prime}\right)$ ), and weaker and smaller components in the western part of the region between $-5-0 \mathrm{~km} \mathrm{~s}^{-1}$ (the maximum $T_{\mathrm{R}}^{*}$ is $2.6 \mathrm{~K}$ at $\left(-16^{\prime}, 16^{\prime}\right)$ ), and between $0-2 \mathrm{~km} \mathrm{~s}^{-1}$ (the maximum $T_{\mathrm{R}}^{*}$ is $1.3 \mathrm{~K}$ at $\left.\left(-52^{\prime},-12^{\prime}\right)\right)$. The offsets of these $T_{\mathrm{R}}^{*}$ maxima differ from peaks in Fig. 1a due to small differences 

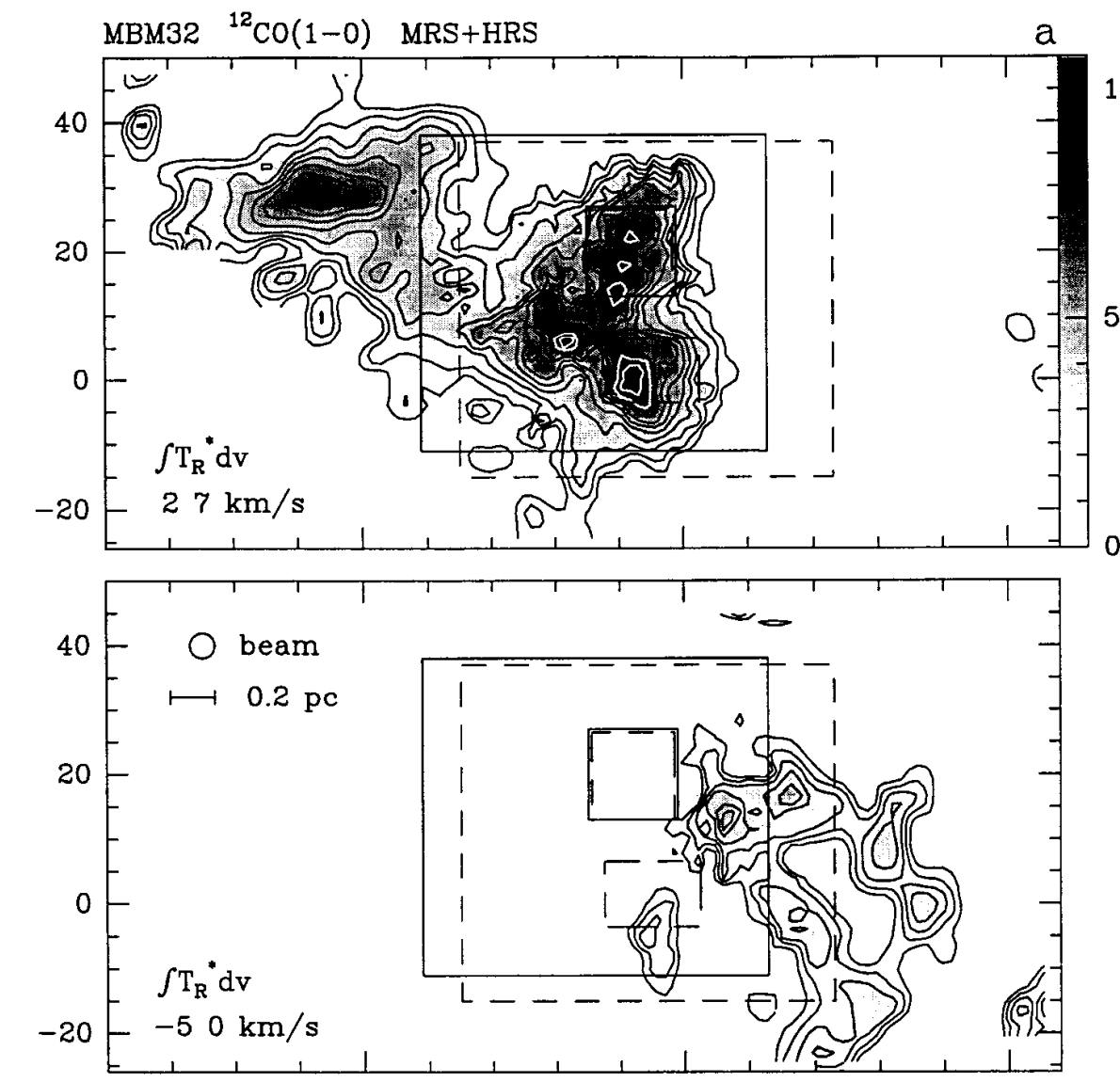

10
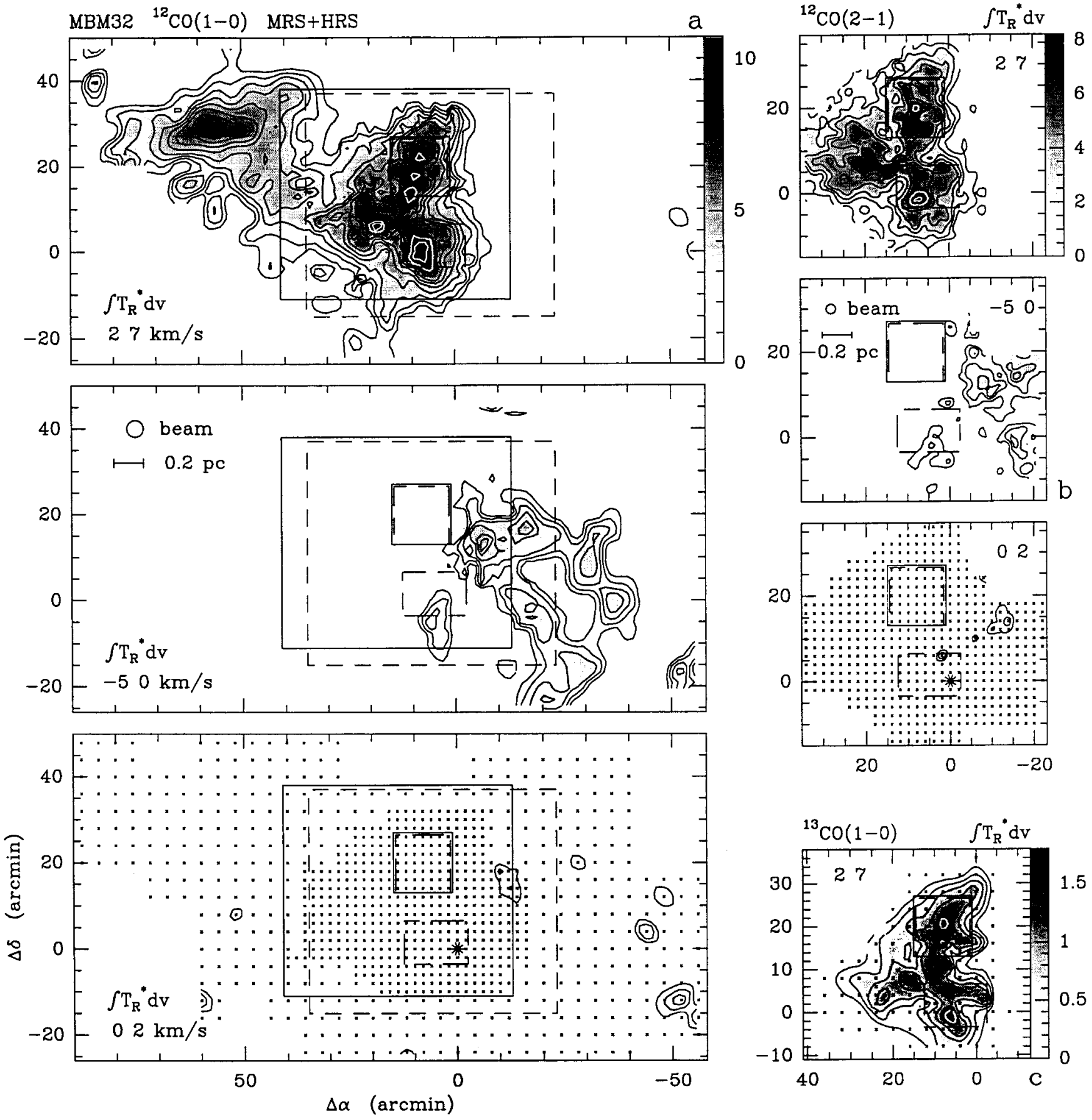

Fig. 1. a) The distribution in MBM 32 of $\int T_{\mathrm{R}}^{*} \mathrm{~d} v$ for ${ }^{12} \mathrm{CO}(J=1-0)$. We combined the $4^{\prime}$ raster MRS and the $2^{\prime}$ raster HRS data. The three panels show the emission in the velocity intervals $2-7 \mathrm{~km} \mathrm{~s}^{-1}$ (top), $-5-0 \mathrm{~km} \mathrm{~s}^{-1}$ (middle), and $0-2 \mathrm{~km} \mathrm{~s}^{-1}$ (bottom). Contour levels are 1 to $10 \mathrm{~K} \mathrm{~km} \mathrm{~s}^{-1}$ in steps of $1 \mathrm{~K} \mathrm{~km} \mathrm{~s}^{-1}$. The small crosses are the observed positions. The asterisk indicates the reference position $\left(0^{\prime}, 0^{\prime}\right)$, see text. The large dashed box is the area mapped in ${ }^{12} \mathrm{CO}(2-1)$ (Fig. 1b), and the large full-drawn box is the area mapped with the HRS in ${ }^{13} \mathrm{CO}(1-0)$ (Fig. 1c). The small full-drawn box is the area mapped in ${ }^{13} \mathrm{CO}(2-1)$ (Fig. 2a) small dashed boxes are the areas mapped in ${ }^{12} \mathrm{CO}(3-2)$ (Figs. 2b,c). b) The same as Fig. 1a, for the ${ }^{12} \mathrm{CO}(J=2-1)$ observations with the MRS made at a $2^{\prime}$ grid spacing in the central area of MBM 32 . Contour levels are 1 to $7 \mathrm{~K} \mathrm{~km} \mathrm{~s}^{-1}$ in steps of $1 \mathrm{~K} \mathrm{~km} \mathrm{~s}^{-1}$. The drawn box in the bottom panel indicates the area observed in ${ }^{13} \mathrm{CO}(2-1)$, and the dashed boxes those observed in ${ }^{12} \mathrm{CO}(3-2)$. c) The same as Fig. $1 \mathrm{~b}$ for the ${ }^{13} \mathrm{CO}(J=1-0)$ observations with the HRS made at a $2-4^{\prime}$ grid spacing in the central area of MBM 32 . Contour levels are 0.25 to $1.5 \mathrm{~K} \mathrm{~km} \mathrm{~s}^{-1}$ in steps of $0.25 \mathrm{~K} \mathrm{~km} \mathrm{~s}^{-1}$ 
Table 1. Parameters of KOSMA observations

\begin{tabular}{|c|c|c|c|c|c|c|}
\hline & \multicolumn{2}{|c|}{${ }^{12} \mathrm{CO}(1-0)$} & ${ }^{13} \mathrm{CO}(1-0)$ & ${ }^{12} \mathrm{CO}(2-1)$ & ${ }^{13} \mathrm{CO}(2-1)$ & ${ }^{12} \mathrm{CO}(3-2)$ \\
\hline$T_{\text {rec }}(\mathrm{K})$ & \multicolumn{2}{|c|}{$355-400$} & $310-360$ & $370-400$ & $400-470$ & $320-350$ \\
\hline$T_{\text {sys }}($ zenith $)(\mathrm{K})$ & \multicolumn{2}{|c|}{$1000-1600$} & $740-820$ & $1000-1800$ & $1200-1800$ & $950-1550$ \\
\hline beamsize $\left({ }^{\prime}\right)$ & \multicolumn{2}{|c|}{3.9} & 3.9 & 2.2 & 2.2 & 1.2 \\
\hline \multirow[t]{2}{*}{$\eta^{\prime}$} & \multicolumn{2}{|c|}{0.76} & 0.76 & 0.67 & 0.67 & $0.52(89 / 90)$ \\
\hline & & & & & & $0.60(91 / 92)$ \\
\hline backend & MRS & HRS & HRS & MRS & MRS & MRS \\
\hline chan. width $\left(\mathrm{km} \mathrm{s}^{-1}\right)$ & 0.43 & 0.082 & 0.086 & 0.21 & 0.22 & 0.14 \\
\hline vel. res. $\left(\mathrm{km} \mathrm{s}^{-1}\right)$ & 0.93 & 0.15 & 0.16 & 0.45 & 0.47 & 0.30 \\
\hline rms noise $\left(T_{\mathrm{R}}^{*}\right)(\mathrm{K})$ & 0.19 & 0.44 & 0.13 & 0.18 & 0.07 & 0.19 \\
\hline \# positions & 555 & 475 & 126 & 540 & 61 & 310 \\
\hline raster $\left({ }^{\prime}\right)$ & 4 & 2 & $2 / 4$ & 2 & 2 & 1 \\
\hline
\end{tabular}

in line widths and noise. The separation between the two smaller components in $\mathrm{CO}$ is less clear in the MRS data east of $\alpha$-offset $-25^{\prime}$, where the smallest component may continue to $-1 \mathrm{~km} \mathrm{~s}^{-1}$. All components show fragments of typically a few resolution elements in size, connected by extended emission. The length of the main component is about 1.6 (2.8 pc).

Because of the relatively narrow lines, the high resolution data show higher $T_{\mathrm{R}}^{*}$ values than the MRS data. Maxima are $T_{\mathrm{R}}^{*}=6.5 \mathrm{~K}$ [at $\left.\left(6^{\prime}, 2^{\prime}\right)\right]$ for the main component, $2.1 \mathrm{~K}$ [at $\left.\left(-12^{\prime}, 14^{\prime}\right)\right]$ for the weak component, and $3.2 \mathrm{~K}\left[\right.$ at $\left.\left(-6^{\prime}, 14^{\prime}\right)\right]$ for the negative velocity component (the MRS peak at $\left(-52^{\prime},-12^{\prime}\right)$ was not observed).

These ${ }^{12} \mathrm{CO}(1-0)$ data can be compared in Figs. 1b and $1 \mathrm{c}$ with the ${ }^{12} \mathrm{CO}(2-1)$ and ${ }^{13} \mathrm{CO}(1-0)$ emission in the same region. ${ }^{12} \mathrm{CO}(2-1)$ peak positions are close to, but not coinciding (probably due to pointing errors and/or noise) with the ${ }^{12} \mathrm{CO}(1-0)$ peaks and the lines are weaker: $T_{\mathrm{R}}^{*}=4.0 \mathrm{~K}$ at $\left(8^{\prime},-2^{\prime}\right)$ for the main component, $1.8 \mathrm{~K}$ at $\left(-14^{\prime}, 14^{\prime}\right)$ for the small component, and $1.9 \mathrm{~K}$ at $\left(-20^{\prime}, 16^{\prime}\right)$ at $V_{\mathrm{lsr}}<0 \mathrm{~km} \mathrm{~s}^{-1}$. In ${ }^{13} \mathrm{CO}(1-0)$ only the main cloud component was detected. It shows two equally strong $(1.6 \mathrm{~K})$ peaks at $\left(0^{\prime}, 4^{\prime}\right)$ and $\left(6^{\prime}, 20^{\prime}\right)$. Only smaller parts of this region were observed in ${ }^{12} \mathrm{CO}(3-2)$ and ${ }^{13} \mathrm{CO}(2-1)$ (see Fig. 2). In ${ }^{12} \mathrm{CO}(3-2)$ the strongest lines are seen within the second ${ }^{13} \mathrm{CO}(1-0)$ peak, which splits into three $2.2 \mathrm{~K}$ peaks at $\left(5^{\prime}, 25^{\prime}\right),\left(8^{\prime}, 18^{\prime}\right)$, and $\left(2^{\prime}, 16^{\prime}\right)$. Within the latter area the strongest ${ }^{13} \mathrm{CO}(2-1)$ emission is near the middle peak $\left[0.7 \mathrm{~K}\right.$ at $\left.\left(9^{\prime}, 17^{\prime}\right)\right]$.

All maps show much structure at all scales, which cannot easily be described. The general structure of the distribution is the same for all transitions, but in the details there are differences, which are possibly caused by excitation effects and/or pointing errors. A quantitative analysis is made in Sect. 4. The position of the two $\mathrm{NH}_{3}$ maxima detected by Heithausen et al. (1998a) or the two $\mathrm{H}_{2} \mathrm{CO}$ maxima found by Heithausen et al. (1987) are in the general region where there are also maxima seen in the different ${ }^{12} \mathrm{CO}$ or ${ }^{13} \mathrm{CO}$ transitions, but there is no clear correlation between the locations of any maximum. This confirms the anticorrelation found by Heithausen et al. (1987) of $\mathrm{H}_{2} \mathrm{CO}$ with lower resolution $\mathrm{CO}$ data. Only a small part of the cloud was observed in either $\mathrm{NH}_{3}$ or $\mathrm{H}_{2} \mathrm{CO}$, and it seems likely that clouds such as MBM 32 contain a large number of such cloudlets. It is unclear whether these cloudlets can form stars. Kun (1992) has searched for $\mathrm{H} \alpha$ emission-line stars towards a sample of high latitude clouds, among them MBM 32. She found three objects with $\mathrm{H} \alpha$ emission which are seen in the direction of the main CO component of MBM 32: K92 27 [at offset $(7 ! 6,-7 ! 5)]$, K92 30 [at offset $(13 ! 5,5 ! 5)$ ], and K92 33 [at offset $(47 ! 4,28 ! 4)$ ]. Since no higher resolution spectra have been made of these stars, it is not yet known whether these objects are indeed $\mathrm{T}$ Tauri stars associated with MBM 32.

\subsection{Velocity structure}

Channel maps in intervals of $1 \mathrm{~km} \mathrm{~s}^{-1}$ of the ${ }^{12} \mathrm{CO}(1-0)$ emission are shown in Fig. 3. The main part of the cloud is at $V_{\mathrm{lsr}}=3-4 \mathrm{~km} \mathrm{~s}^{-1}$, but both the central and eastern maxima show small velocity gradients. Gaussfits show that the velocities of the line peaks range from -4.0 to $5.3 \mathrm{~km} \mathrm{~s}^{-1}$ for the MRS ${ }^{12} \mathrm{CO}(1-0)$ data.

The other transitions have not been mapped in the whole area of Fig. 1a and therefore show emission in a smaller velocity range. For the central region the velocity structure is shown in more detail in Fig. 4 for ${ }^{12} \mathrm{CO}(2-1)$. The channel maps for the two small areas observed in ${ }^{12} \mathrm{CO}(3-2)$ and in ${ }^{13} \mathrm{CO}(2-1)$ (not shown) show structures which agree with those visible in the $1^{\prime}$ resolution ${ }^{12} \mathrm{CO}(1-0)$ map of a small $\left(8^{\prime} \times 16^{\prime}\right)$ region by Magnani et al. (1990).

At most positions the CO line shapes are Gaussian within the noise and show no structure such as self absorption or wings. However there are some exceptions. This is best seen in the higher resolution ${ }^{12} \mathrm{CO}(3-2)$ spectra: the main cloud shows at offsets around $\left(7^{\prime}, 21^{\prime}\right)$ a stronger line at $3.5 \mathrm{~km} \mathrm{~s}^{-1}$ and weaker emission at about $2.0 \mathrm{~km} \mathrm{~s}^{-1}$. 

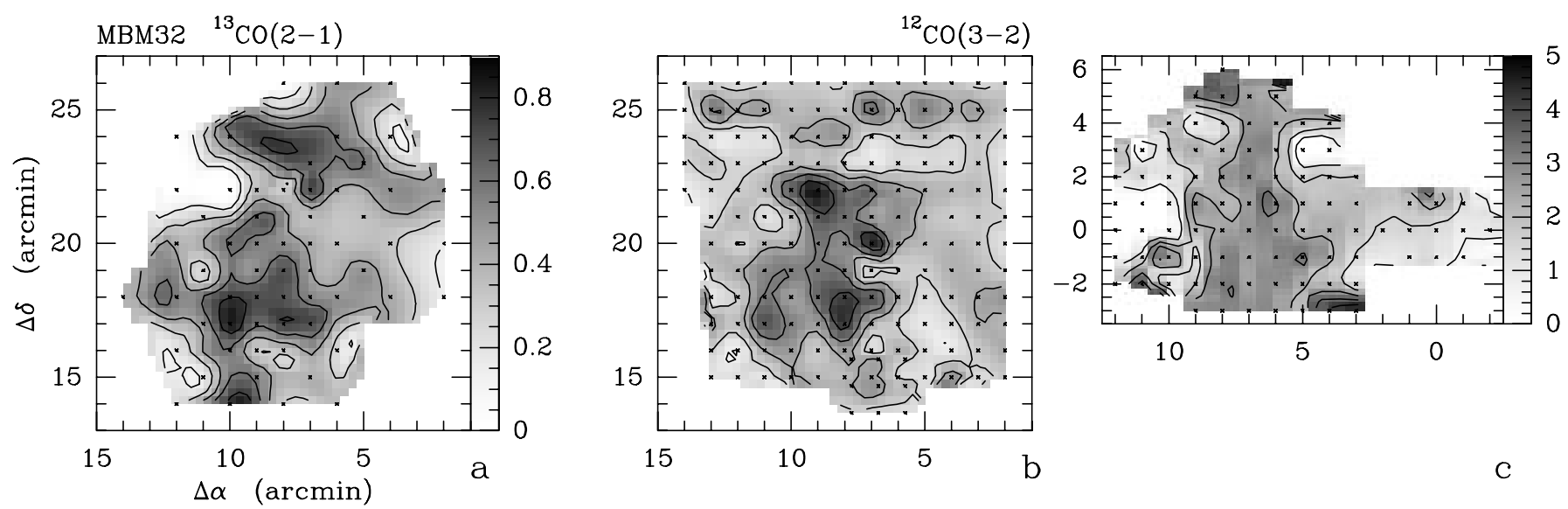

Fig. 2. The same as Fig. 1, for a) the ${ }^{13} \mathrm{CO}(J=2-1)$ observations with the MRS made at a $1-2^{\prime}$ grid spacing in the central area of MBM 32. Contour levels are 0.15 to $0.75 \mathrm{~K} \mathrm{~km} \mathrm{~s}^{-1}$ in steps of $0.15 \mathrm{~K} \mathrm{~km} \mathrm{~s}^{-1}$. b,c) the ${ }^{12} \mathrm{CO}(J=3-2)$ emission in the interval $2-7 \mathrm{~km} \mathrm{~s}^{-1}$ obtained with the MRS at a $1^{\prime}$ grid spacing in two regions in the central area of MBM 32 . Contour levels are 0.25 to $1.5 \mathrm{~K} \mathrm{~km} \mathrm{~s}^{-1}$ in steps of $0.25 \mathrm{~K} \mathrm{~km} \mathrm{~s}^{-1}$

Northeast of this position the line shapes are asymmetric. We explain this by overlapping in this region of the two fragments of the main cloud of MBM 32 (see Fig. 4).

The line widths of the negative velocity cloud (and possibly also of the weak emission in the interval $0-2 \mathrm{~km} \mathrm{~s}^{-1}$ ) are somewhat larger than those of the main cloud. This is consistent with the findings of de Vries et al. (1987). Within the main cloud, line widths at the west side $\left(\Delta \alpha<+6^{\prime}\right)$ are significantly smaller than in the rest of the cloud. This is seen in all transitions, but the effect depends on the velocity resolution of the different spectra. The $115 \mathrm{GHz}$ HRS data show an average value of $1.12 \pm 0.26 \mathrm{~km} \mathrm{~s}^{-1}$ (135 positions) in the western part and of $1.49 \pm 0.33$ (249 positions) in the eastern part. This division is not clearly correlated with some clumps or peaks in the cloud. The negative velocity emission has an average line width of $1.92 \pm 0.63 \mathrm{~km} \mathrm{~s}^{-1}$ (108 positions). Except for the region NE of $\left(7^{\prime}, 21^{\prime}\right)$ (see above), the differences in line widths cannot be explained by the presence of more than one velocity component along the line of sight.

\subsection{H I}

The neutral hydrogen emission towards MBM 32 might be confused by an unknown amount due to fore- and background components at about the same velocity as the CO. However, Vladilo et al. (1994) concluded from optical data that in the direction of (among others) MBM 32 the local bubble extends to the assumed distance of MBM 32 $(100 \mathrm{pc})$. If this is true, the amount of $\mathrm{HI}$ foreground emission will be low. Background emission was also found at velocities considerably different from that of MBM 32. The strongest $\mathrm{HI}$ lines found in the mapped region are $31.8 \mathrm{~K}$ at offset $\left(88^{\prime}, 12^{\prime}\right)$, at the eastern border of MBM 32 . This emission is at $2.9 \mathrm{~km} \mathrm{~s}^{-1}$, near the velocity of the molec- ular cloud emission. The weakest line near the velocity of the main $\mathrm{CO}$ component is $7.2 \mathrm{~K}\left(\right.$ at $\left.4.7 \mathrm{~km} \mathrm{~s}^{-1}\right)$ at $\left(-16^{\prime}\right.$, $\left.16^{\prime}\right)$, in the region where is the negative velocity component of CO. Stronger HI emission at negative velocities is usually due to the wings of the main component emission. The weakest line at negative velocities $(7.1 \mathrm{~K})$ is at $\left(-20^{\prime}\right.$, $\left.40^{\prime}\right)$, north of the corresponding $\mathrm{CO}$ line, and at a minimum in the IRAS 60 and $100 \mu \mathrm{m}$ maps (see Sect. 3.4). To compare $\mathrm{CO}$ and $\mathrm{HI}$ emission for MBM 32 we have plotted in Fig. 5 the $\mathrm{HI}$ and ${ }^{12} \mathrm{CO}(1-0)$ spectra, summed for all positions where we detected ${ }^{12} \mathrm{CO}(1-0)$. For the main $\mathrm{CO}$ component we distinguish between the two largest cloud fragments, east and west of $\Delta \alpha=+34^{\prime}$. It is seen that in each of the Figs. 5a to d, the Hi spectra have a peak close to the $\mathrm{CO}$ velocity. Except for the smallest $\mathrm{CO}$ component (Fig. 5b), it is also the strongest feature in each $\mathrm{HI}$ spectrum, which in addition contains emission from both other components. In each spectrum there is also a very broad $\left(>15 \mathrm{~km} \mathrm{~s}^{-1}\right)$ component, which is due to warmer, unrelated gas.

Because the Hi emission has a larger velocity range and intrinsic line width than the CO emission (see Fig. 5), and shows small differences in velocity compared to the $\mathrm{CO}$ (see below), it is not much of use to integrate the HI emission over the same velocity interval as the $\mathrm{CO}$ or to investigate the peak intensities. Instead we show in Fig. 6 the channel maps with intervals of $2 \mathrm{~km} \mathrm{~s}^{-1}$ between -8 and $+10 \mathrm{~km} \mathrm{~s}^{-1}$. HI at negative velocities is mainly present in the western part of the cloud where there is also $\mathrm{CO}$ emission at $V_{\mathrm{lsr}}<0 \mathrm{~km} \mathrm{~s}^{-1}$. The main part of the Hi emission is in the interval +1 to $+4 \mathrm{~km} \mathrm{~s}^{-1}$ where there is some correlation of the HI distribution with that of the strongest $\mathrm{CO}$ component. The strongest HI is just NE of the eastern $\mathrm{CO}$ peak. The negative velocity $\mathrm{CO}$ component, which it's approximately elliptical distribution shows a similar 

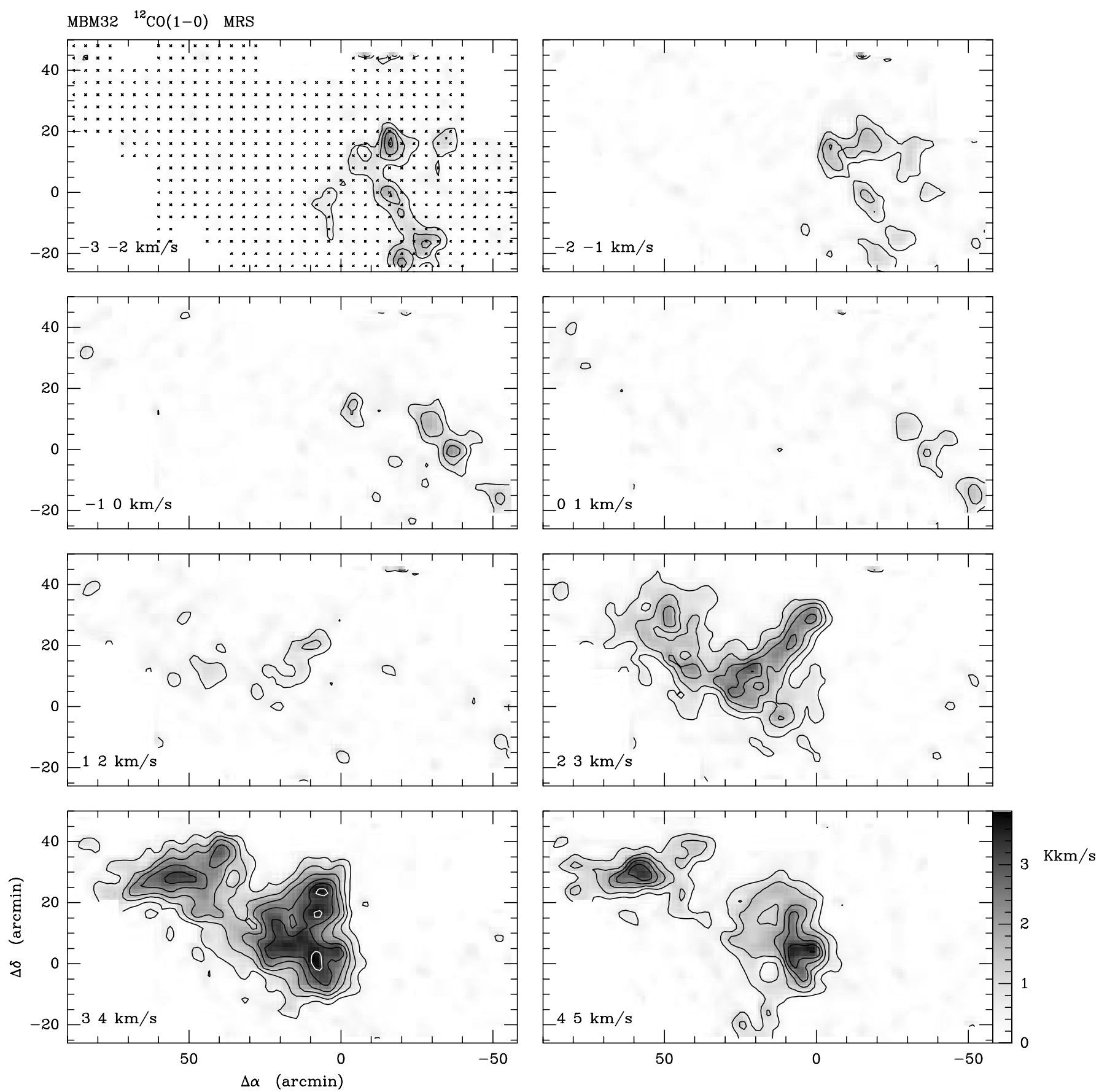

Fig. 3. Channel maps in intervals of $1 \mathrm{~km} \mathrm{~s}^{-1}$ of the ${ }^{12} \mathrm{CO}(1-0)$ emission in MBM 32 observed with the MRS at a $4^{\prime}$ grid. Contour levels are 0.6 to $3.6 \mathrm{~K} \mathrm{~km} \mathrm{~s}^{-1}$ in steps of $0.6 \mathrm{~K} \mathrm{~km} \mathrm{~s}^{-1}$. The small crosses are the observed positions

structure in $\mathrm{HI}$ at -4 to $-2 \mathrm{~km} \mathrm{~s}^{-1}$ and -2 to $0 \mathrm{~km} \mathrm{~s}^{-1}$ which seems to be slightly outside the $\mathrm{CO}$ emission region.

The velocity structure of the Hi gas associated with MBM 32 is shown from another perspective in Fig. 7, which contains position $(\Delta \alpha)$ - velocity diagrams at $\delta$ offsets from $-24^{\prime}$ to $+44^{\prime}$. The velocity components from Fig. 5 are clearly visible, along with many other structures, such as secondary maxima, holes, and velocity gradients, which cannot easily be modeled.
We have made Gaussian fits to the Hi spectra using two to four velocity components: one weak (few K) and broad (typically $20-40 \mathrm{~km} \mathrm{~s}^{-1}$ ) component and stronger but narrower $\left(<10 \mathrm{~km} \mathrm{~s}^{-1}\right)$ components. The broad component shows little variation in intensity over the mapped region and we assume that it is not associated with MBM 32. In Fig. 8 we compare the velocities of the narrow components with the $V_{\mathrm{lsr}}$ of the MRS+HRS ${ }^{12} \mathrm{CO}(1-0)$ data. Only in part of the main CO cloud $\left(-5^{\prime}<\Delta \alpha<37^{\prime}\right)$ 

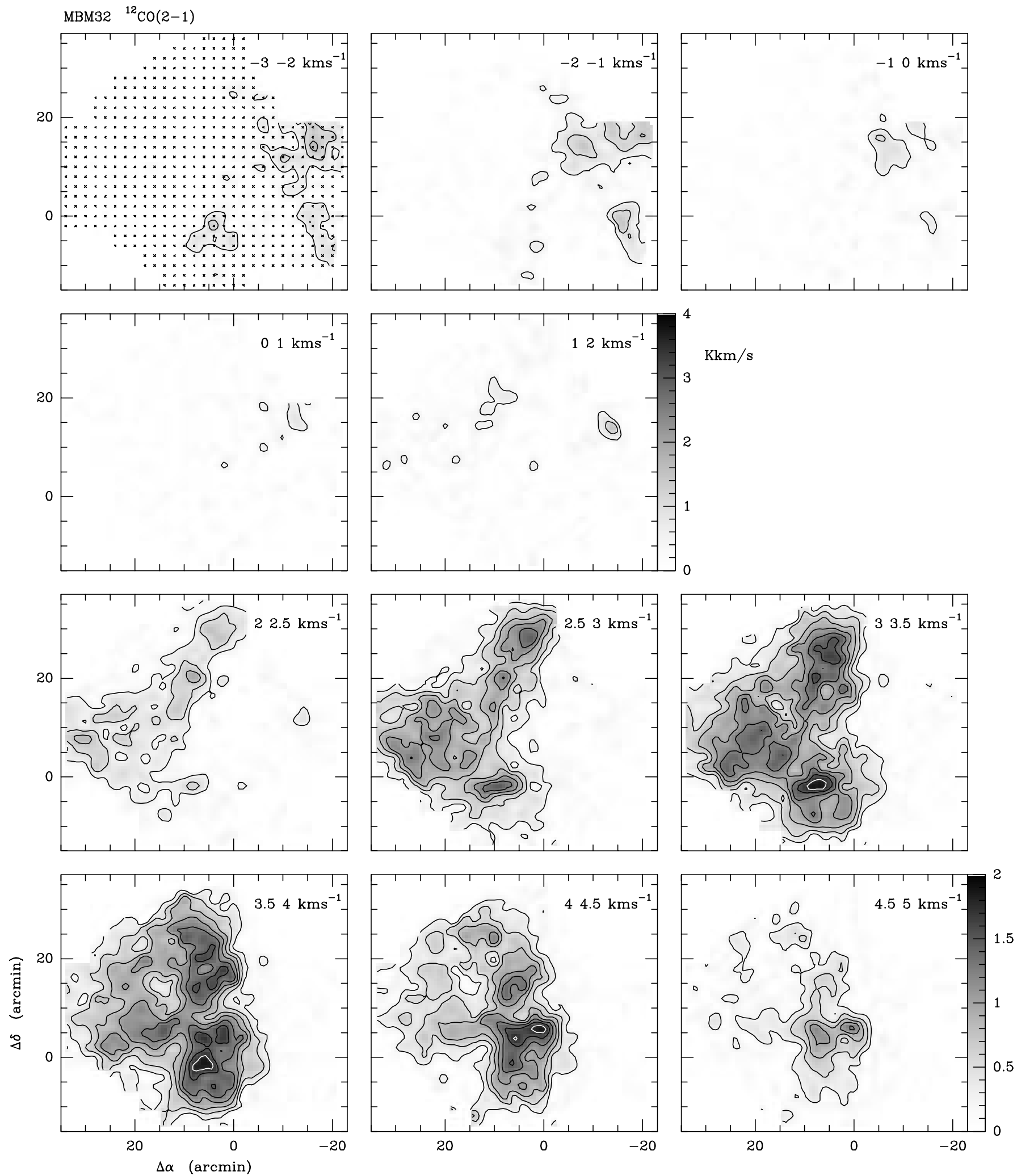

Fig. 4. The same as Fig. 3 for the ${ }^{12} \mathrm{CO}(2-1)$ emission in the central part of MBM 32 observed with the MRS at a $2^{\prime}$ grid. Note that the velocity step used changes at $V_{\mathrm{lsr}} 2 \mathrm{~km} \mathrm{~s}^{-1}$. Contour levels are 0.5 to $2 \mathrm{~K} \mathrm{~km} \mathrm{~s}^{-1}$ in steps of $0.5 \mathrm{~K} \mathrm{~km} \mathrm{~s}^{-1}$ $\left(V_{\mathrm{lsr}}<2 \mathrm{~km} \mathrm{~s}^{-1}\right)$ and 0.25 to $2 \mathrm{~K} \mathrm{~km} \mathrm{~s}^{-1}$ in steps of $0.25 \mathrm{~K} \mathrm{~km} \mathrm{~s}^{-1}\left(V_{\mathrm{lsr}}>2 \mathrm{~km} \mathrm{~s}^{-1}\right)$ 

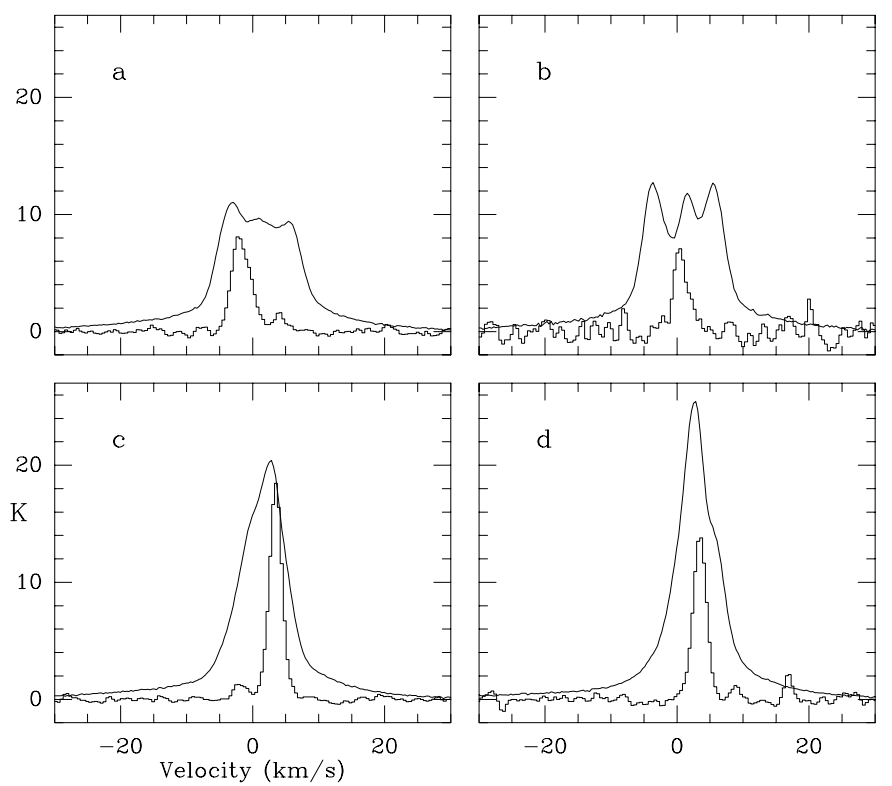

Fig. 5. Comparison of $\mathrm{HI}$ (full drawn) and ${ }^{12} \mathrm{CO}(1-0)$ (MRS; histograms) emission. All Hi spectra (the broader lines) are on the same $\left(T_{B}\right)$ scale. The spectra were averaged for positions where there is $\mathrm{CO}$ emission with $V_{\mathrm{lsr}}<0 \mathrm{~km} \mathrm{~s}^{-1}$ a), $0<V_{\mathrm{lsr}}<2 \mathrm{~km} \mathrm{~s}^{-1}$ b), $V_{\mathrm{lsr}}>2 \mathrm{~km} \mathrm{~s}^{-1}$ at $\Delta \alpha<34^{\prime}$ c), or $\left.>34^{\prime} \mathbf{d}\right)$. The $\mathrm{CO}$ intensities $\left(T_{\mathrm{R}}^{*}\right)$ were multiplied by a factor of 10

the velocities of one of the Hi components agrees with the $\mathrm{CO}$ velocities within $0.5 \mathrm{~km} \mathrm{~s}^{-1}$. For the other CO components differences are in the range $-1.5-+1.5 \mathrm{~km} \mathrm{~s}^{-1}$. At positions where there are such differences, the Hi spectra do not show signs of self absorption due to cold foreground gas. These differences are less than the velocity dispersion of the molecular gas: the line widths of spectra of the sum of all ${ }^{12} \mathrm{CO}(1-0)$ MRS data are $2.4 \mathrm{~km} \mathrm{~s}^{-1}$ (main component) and $3.4 \mathrm{kms}^{-1}$ (negative velocity component). It is possible that only the HI gas in a certain velocity range is converted into $\mathrm{H}_{2}$, leaving stronger emission at higher or lower velocities. Or HI at one edge of the cloud has a different velocity due to external pressure. Hi line widths of all components are about $4-5 \mathrm{~km} \mathrm{~s}^{-1}$ : the average value for the component associated with the main $\mathrm{CO}$ cloud is $4.95 \pm 1.98 \mathrm{~km} \mathrm{~s}^{-1}$; those of the three components at negative offsets are $4.35 \pm 0.91 \mathrm{~km} \mathrm{~s}^{-1}\left(V_{\mathrm{lsr}}<\right.$ $\left.0 \mathrm{~km} \mathrm{~s}^{-1}\right), 4.75 \pm 1.75 \mathrm{~km} \mathrm{~s}^{-1}\left(0<V_{\mathrm{lsr}}<4 \mathrm{~km} \mathrm{~s}^{-1}\right)$, and $4.31 \pm 1.20 \mathrm{~km} \mathrm{~s}^{-1}\left(V_{\mathrm{lsr}}>4 \mathrm{~km} \mathrm{~s}^{-1}\right)$. These values are a factor 2-4 larger than the CO line widths in the same region, which is less than expected from the difference in mass of HI and CO. It suggests that either the molecular gas is relatively more turbulent, or the Hi gas is colder. We have used the Hi data to derive masses assuming the emission is optically thin $\left[N(\mathrm{HI})=1.82210^{18} \int T_{B} \mathrm{~d} v \mathrm{~cm}^{-2}\right]$. The resulting values (not including $\mathrm{He}$ ) are indicated in Fig. 8 for the different velocity components. We also indicate the $\mathrm{H}_{2}$ masses derived from $\mathrm{CO}$ (see Sect. 4.1). If all HI is associated with MBM 32, both masses are approxi- mately equal for the main $\mathrm{CO}$ component. For the negative velocity component, the $\mathrm{H}_{2}$ mass is somewhat larger than the Hi mass (however this depends which region is considered).

\subsection{FIR emission}

The distribution of the dust emission associated with MBM 32 can be seen in the 60 and $100 \mu \mathrm{m}$ IRAS (ISSA) maps in Figs. 9a and b, which have an angular resolution of about $5^{\prime}$. For comparison the integrated ${ }^{12} \mathrm{CO}(1-0)$ and $\mathrm{HI}$ distribution are shown in Figs. 9c and $\mathrm{d}$ on the same scale. The IRAS data are the maps produced from all HCONs, and were taken from the Sky Survey Atlas. To avoid negative values of flux densities in the $60 \mu \mathrm{m}$ map we added here a constant offset to all pixels of $1 \mathrm{MJy} / \mathrm{sr}$. There clearly is FIR emission associated with the molecular clouds, both at 60 and $100 \mu \mathrm{m}$. However the distribution is confused by emission from dust associated with the HI gas, which has a slightly different distribution. The Hi maximum near offset $\left(50^{\prime}, 40^{\prime}\right)$ outside the $\mathrm{CO}$ cloud shows as a peak in the $60 \mu \mathrm{m}$ map suggesting the presence of relatively warm dust in this region. A combined image of 12,60 , and $100 \mu \mathrm{m}$ emission in MBM 32 was produced also by Verter \& Rickard (1998), however without interpretation.

Before we can compare the CO, HI, and FIR emission, we have to convolve the CO and IRAS 60 and $100 \mu \mathrm{m}$ data first to the same resolution as the Hi $(9 ! 2)$ data. Here we use only the ${ }^{12} \mathrm{CO}(1-0)$ MRS map because it covers the largest area on the sky. Subsequently we sampled the FIR data at the same $\left(4^{\prime}\right)$ raster as both other data sets. The correlation between FIR and HI emission was investigated by selecting positions where the convolved $\mathrm{CO}$ spectra showed no emission stronger than about $0.2 \mathrm{~K}$ (114 positions). We compared the $100 \mu \mathrm{m}$ emission with the $\mathrm{HI}$ emission integrated over the whole velocity range where there is emission ( -80 to $50 \mathrm{~km} \mathrm{~s}^{-1}$ ), with the HI emission of the narrow components in the Gaussfits, and with all the local emission (including the broad component). It appears (using the bisector linear least squares fit, see Isobe et al. 1990) that in the latter case the correlation is best (correlation coefficient 0.60 ), however the offset remains rather uncertain:

$F_{100 \mu \mathrm{m}}=(-3.438 \pm 0.524)+(0.034 \pm 0.003) \int T_{\mathrm{b}}(\mathrm{HI}) \mathrm{d} v$.

Here, $F_{100 \mu \mathrm{m}}$ is the flux density in $\mathrm{MJy} \mathrm{sr}^{-1}$. For $60 \mu \mathrm{m}$ the correlation coefficient is 0.49 :

$F_{60 \mu \mathrm{m}}=(-1.545 \pm 0.094)+(0.0059 \pm 0.0006) \int T_{\mathrm{b}}(\mathrm{HI}) \mathrm{d} v$.

The correlation for $100 \mu \mathrm{m}$ (see Fig. 10a) is better than found by Meyerdierks \& Heithausen (1996) for the HLCs of the Polaris Flare, because in the case of MBM 32 there 

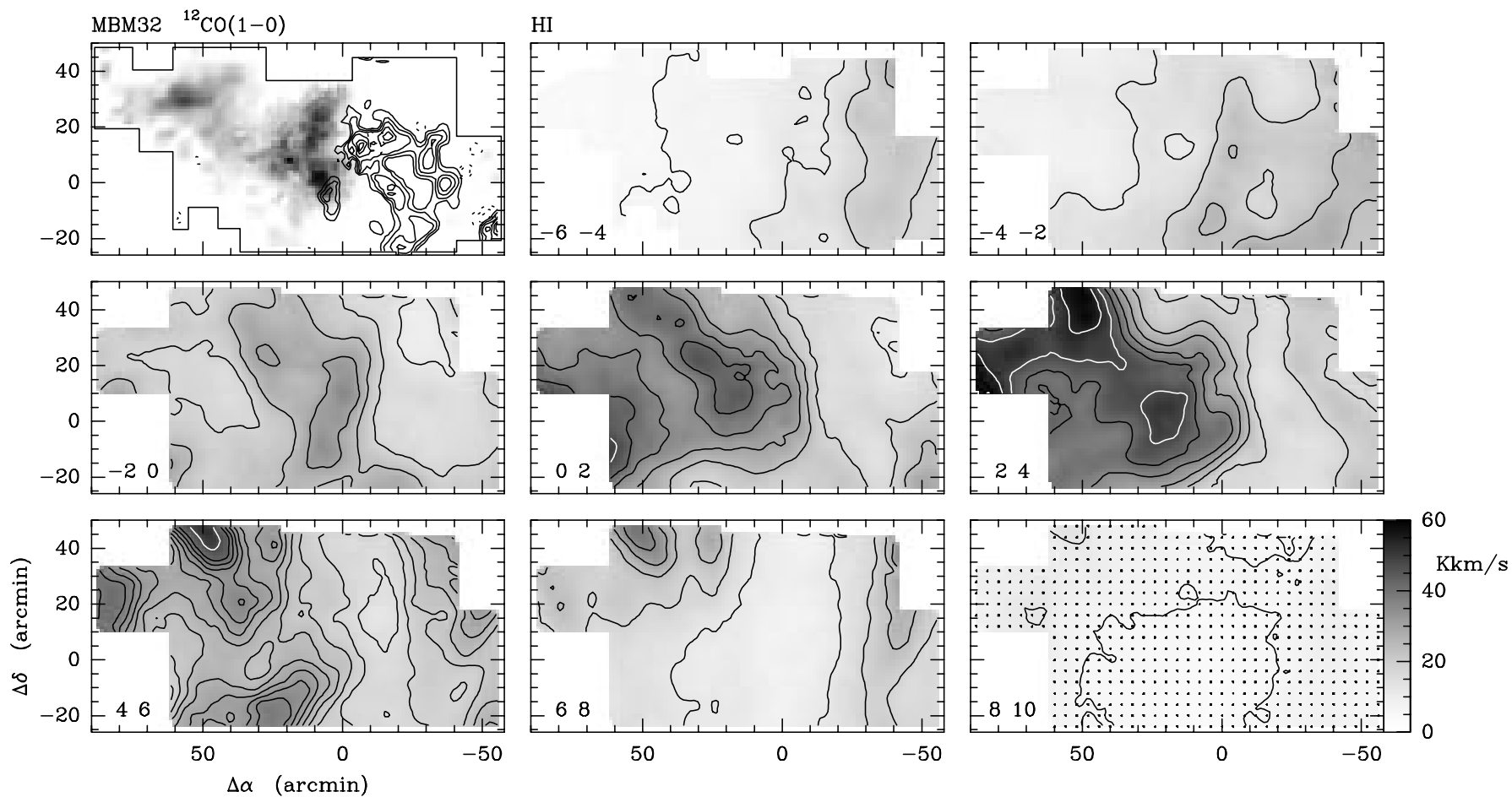

Fig. 6. Channel maps of Hi emission in intervals of $2 \mathrm{~km} \mathrm{~s}^{-1}$ between -8 and $10 \mathrm{~km} \mathrm{~s}^{-1}$. Contour levels are 6 to $60 \mathrm{~K} \mathrm{~km} \mathrm{~s}{ }^{-1}$ in steps of $6 \mathrm{~K} \mathrm{~km} \mathrm{~s}^{-1}$. For comparison in the upper left panel the ${ }^{12} \mathrm{CO}(1-0)$ distribution for the three velocity intervals from Fig. 1, as grey-scale (Fig. 1s), full-drawn (Fig. 1b), and as dotted (Fig. 1c) contours

are no other branches with different slope, probably because towards the Polaris Flare a larger area of the sky was investigated containing regions with different properties. There is no significant difference in Figs. 10a and $\mathrm{b}$ between the distribution of the points located in the eastern (negative $\mathrm{CO}$ velocity) and western cloud parts.

The results (Eq. 1) were used to correct the FIR emission for dust associated with HI towards the 403 positions where there is CO emission, and subsequently we obtained the following relation between $100 \mu \mathrm{m}$ emission and ${ }^{12} \mathrm{CO}(1-0)$ integrated line intensities:

$\Delta F_{100 \mu \mathrm{m}}=(-0.737 \pm 0.062)+(0.618 \pm 0.021) \int T_{\mathrm{R}}^{*}(1-0) \mathrm{d} v$.

The correlation coefficient is 0.73 . We distinguished between three parts of the cloud and obtained the following slopes: $0.857 \pm 0.046$ (for $\Delta \alpha<-10^{\prime}$ ), $0.601 \pm 0.029$ (for $-10^{\prime}<\Delta \alpha<34^{\prime}$ ), and $0.596 \pm 0.028$ (for $\Delta \alpha>34^{\prime}$ ). In the latter case the correlation coefficient is best $(0.79)$. These results are shown in Fig. 10c. The range in CO intensity is smaller for the eastern cloud part (containing the negative velocity components), where the slope is steepest.

For $60 \mu \mathrm{m}$ our result is (correlation coefficient 0.60 ):

$F_{60 \mu \mathrm{m}}=(-0.144 \pm 0.011)+(0.088 \pm 0.004) \int T_{\mathrm{R}}^{*}(1-0) \mathrm{d} v$.
For the negative velocity CO component $\left(\Delta \alpha<-10^{\prime}\right)$ there is little correlation. The slope does not change when using only data with $\Delta \alpha>-10^{\prime}: 0.086 \pm 0.004$. The results are shown in Fig. 10d.

The slope in Eq. (1a) translates into a ratio of HI column density and $100 \mu \mathrm{m}$ flux density of $N(\mathrm{H}) / F_{100 \mu \mathrm{m}}=$ $5.410^{19} \mathrm{~cm}^{-2} \mathrm{Jy}^{-1}$ sr. Comparing this with Eq. (2) of Meyerdierks \& Heithausen (1996) we find that the $100 \mu \mathrm{m}$ emission per $\mathrm{H}$ atom is a factor 2.6 smaller in MBM 32 than in the Polaris Flare.

The slope in Eq. (2a), 0.618 MJy s $\left(\mathrm{K} \mathrm{km} \mathrm{sr}^{-1}\right.$ is slightly steeper than the value found by Meyerdierks $\&$ Heithausen $\left[0.5\right.$ MJy s $\left.(\mathrm{K} \mathrm{km} \mathrm{sr})^{-1}\right]$. However their Fig. 7 essentially shows a scatterplot and no accurate value for the slope could be determined. From Eqs. (1a) and $(2 \mathrm{a})$ we obtain a conversion factor between $W_{\mathrm{CO}}(=$ $\int T \mathrm{~d} v\left({ }^{12} \mathrm{CO}(1-0)\right)$ and $N\left(\mathrm{H}_{2}\right)$ of $(0.17 \pm 0.02) 10^{20} \mathrm{~cm}^{-2}$ $\left(\mathrm{K} \mathrm{km} \mathrm{s}^{-1}\right)^{-1}$, much lower than derived from our $\mathrm{CO}$ data alone $\left(0.710^{20}\right.$; see Sect. 4.1), but similar to values derived in the same way towards some parts of the Draco nebula by Herbstmeier et al. (1993). However, this value is a lower limit (possibly by a factor 2 or 3 ) if the dust temperature is lower in the inner parts of the cloud (see Meyerdierks \& Heithausen 1996).

Our results can also be used to derive the dust temperature and mass. A correlation between 60 and $100 \mu \mathrm{m}$ emission associated with MBM 32 gives (correlation 

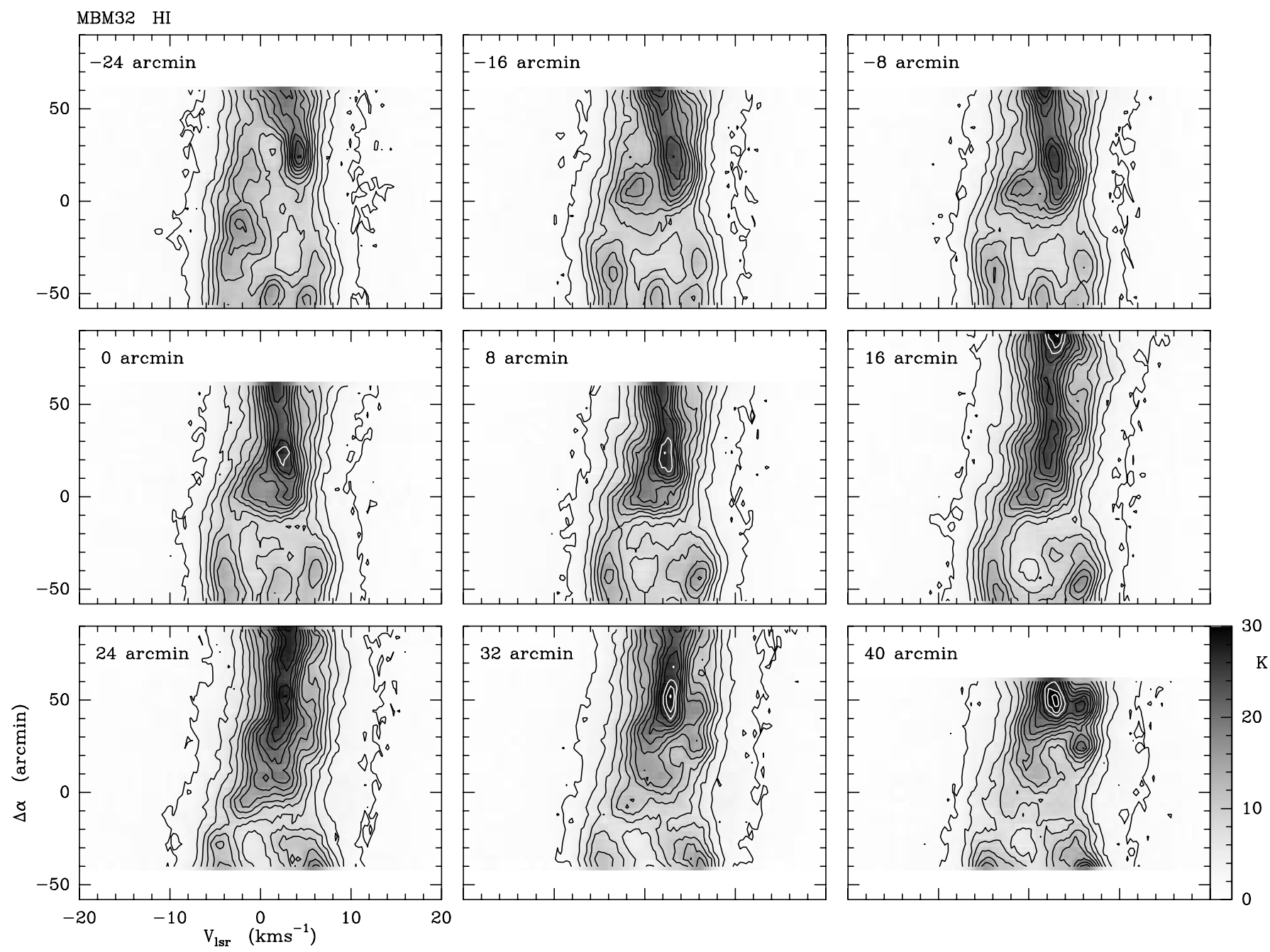

Fig. 7. Position (Right Ascension offset) - velocity diagrams of Hi emission at different $\delta$-offsets (indicated in the panels). Offsets are with respect to $\alpha(1950)=9^{\mathrm{h}} 28^{\mathrm{m}} 42^{\mathrm{s}}, \delta(1950)=+66^{\circ} 5^{\prime}$. Contour levels are 2 to $30 \mathrm{~K}$ in steps of $2 \mathrm{~K}$

coefficient 0.92):

$$
\Delta F_{100 \mu \mathrm{m}}=(0.200 \pm 0.026)+(7.840 \pm 0.132) \Delta F_{60 \mu \mathrm{m}}
$$

The offset is mainly caused by the uncertain zero point in the subtraction of dust associated with background Hi gas. From the slope in Eq. (3), we derive a dust temperature of $20 \mathrm{~K}$, assuming a dust emissivity proportionally to $\lambda^{-2}$. The data are consistent with a constant dust temperature in the cloud which does not depend on CO intensity. To derive the mass of the dust associated with the $\mathrm{CO}$ from the $100 \mu \mathrm{m}$ data (using the method outlined by Wood et al. 1994), we have to correct the latter by a remaining offset. The value of $0.200 \mathrm{MJy} \mathrm{sr}^{-1}$ from Eq. (3) is probably not correct because there might be in addition a remaining offset in the $60 \mu \mathrm{m}$ emission. Instead we choose the value from Eq. (2a), (-0.737), giving the amount of dust associated with $\mathrm{CO}$ (there could be additional dust around the $\mathrm{CO}$ cloud). We used no lower limit for the optical depth at $100 \mu \mathrm{m}$. The total dust mass then is $0.073 \pm 0.022 M_{\odot}$, where the error is determined by an uncertainty in the offset of $0.5 \mathrm{MJy} \mathrm{sr}^{-1}$. The dust mass in the western part of MBM $32\left(\Delta \alpha<-10^{\prime}\right)$ is $0.020 M_{\odot}$. The maximum optical depth at $100 \mu \mathrm{m}$ is $0.23 \mathrm{~m}$ Neper (corresponding to $A_{V}=4.6 \mathrm{mag}$, using Eq. (5) from Wood et al. 1994), at offset $\left(8^{\prime}, 16^{\prime}\right)$. This value appears relatively high compared to optically derived values (0.6 mag; see Sect. 1$)$. The $A_{V}$, derived from the cloud-averaged $100 \mu \mathrm{m}$ flux density, is $1.49 \mathrm{mag}$, which is also higher than the optical values. The ratio of gas mass (derived from the $\mathrm{CO}$ data, see Sect. 4.1: $\left.21.5 M_{\odot}\right)$, and dust mass $\left(0.076 M_{\odot}\right)$ is 283 , which is higher than the usually adopted values of 100 , and suggests that part of the dust is colder than $20 \mathrm{~K}$. However the fraction of such cold dust is less than in most other molecular clouds, where gas to dust ratios above 1000 are found (Wood et al. 1994). Our data of MBM 32 do not suggest the presence in this cloud of an infrared excess such as found by Meyerdierks \& Heithausen (1996) in the Polaris Flare (interpreted as $\mathrm{H}_{2}$ gas without detected $\mathrm{CO})$. 

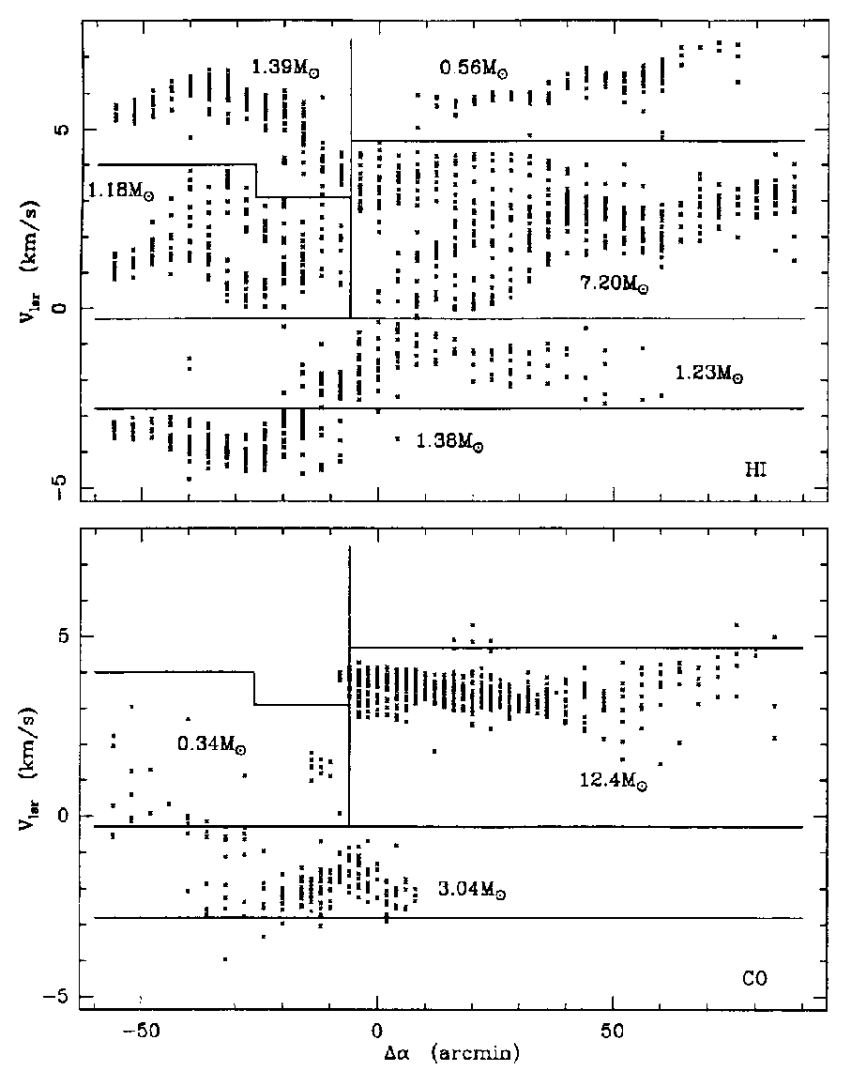

Fig. 8. $V_{\text {lsr }}$ plotted against Right Ascension offset (with respect to $\left.\alpha(1950)=9^{\mathrm{h}} 28^{\mathrm{m}} 42^{\mathrm{s}}\right)$ using velocities from Gaussian fits to Hi profiles (only narrow components) and ${ }^{12} \mathrm{CO}(1-0)$ data (lower panel). Masses (without He contribution) for different velocity components are indicated

\section{CO column densities and excitation}

\subsection{Column densities}

High-latitude clouds such as MBM 32 differ from dark clouds and GMC's by low extinction and low (column) densities, and possibly subthermal excitation of CO. Consequently, ${ }^{12} \mathrm{CO}$ transitions might not be optically very thick at all positions in contrast to dark clouds. Thus the standard methods for deriving physical parameters from $\mathrm{CO}(1-0)$ data will not be quite correct. However, because the magnitude of the error due to these excitation conditions is not known, we shall derive column densities using the standard LTE method from ${ }^{12} \mathrm{CO}$ and ${ }^{13} \mathrm{CO}(1-0)$ data, and then discuss these results. Other methods for deriving $\mathrm{CO}$ (or $\mathrm{H}_{2}$ ) column densities use the integrated ${ }^{12} \mathrm{CO}(1-0)$ intensity $\left(W_{\mathrm{CO}}\right)$, by adopting either standard conversion factors, or by calibrating the data by combining $\mathrm{CO}$, HI, and FIR measurements (see e.g. Magnani \& Onello 1995, for a discussion of these methods).

To derive LTE column densities from the ${ }^{12} \mathrm{CO}$ and ${ }^{13} \mathrm{CO}(1-0)$ data, we follow the method outlined by e.g.
Brand \& Wouterloot (1998), and assume for the ${ }^{13} \mathrm{CO}$ abundance the value of $2.010^{-6}$ derived by Dickman (1978).

The highest value for the $\mathrm{H}_{2}$ column density in MBM 32 that we derive is $8.910^{20} \mathrm{~cm}^{-2}$ at offset $\left(8^{\prime}, 20^{\prime}\right)$. The ratio ${ }^{12} \mathrm{CO} /{ }^{13} \mathrm{CO}(1-0)$ increases with $T_{\mathrm{R}}^{*}\left({ }^{12} \mathrm{CO}(1-0)\right)$ from $5.2(>5 \mathrm{~K})$ to $6.9(2-3 \mathrm{~K})$, suggesting a decrease in optical depth from the cloud center to the edge. Due to the smaller ${ }^{13} \mathrm{CO}$ line widths the integrated $T_{\mathrm{R}}^{*}$ ratio increases from 6.9 to 11.1 in this interval. These ratios are higher than those found in Giant Molecular Clouds, where they are typically $3-5$. We cannot directly derive column densities towards all positions because ${ }^{13} \mathrm{CO}(1-0)$ was not measured at all positions where we have ${ }^{12} \mathrm{CO}(1-0)$ HRS data, and therefore we first compared for those positions where this is possible, $N\left(\mathrm{H}_{2}\right)_{\text {LTE }}$ and $W_{\mathrm{CO}}$ (see Fig. 11). It is seen that the ratio $X^{\prime}=N\left(\mathrm{H}_{2}\right)_{\mathrm{LTE}} / W_{\mathrm{CO}}$ is independent of $W_{\mathrm{CO}}$ (though with a large spread) at a value of $7.0 \pm 2.5$ (s.d.) $10^{19} \mathrm{~cm}^{-2}\left(\mathrm{~K} \mathrm{~km} \mathrm{~s}^{-1}\right)^{-1}$. To derive cloud masses we use this value, rather than the value of $1.910^{20} \mathrm{~cm}^{-2}$ $\left(\mathrm{K} \mathrm{km} \mathrm{s}^{-1}\right)^{-1}$ (Strong \& Mattox 1996) or $1.610^{20} \mathrm{~cm}^{-2}$ $\left(\mathrm{K} \mathrm{km} \mathrm{s}^{-1}\right)^{-1}$ (Hunter et al. 1997) found for inner Galaxy clouds. But it is close to the number derived from gammaray data by Digel et al. (1996) for the Polaris Flare, $0.92 \pm 0.1410^{20} \mathrm{~cm}^{-2}\left(\mathrm{~K} \mathrm{~km} \mathrm{~s}^{-1}\right)^{-1}$. Similarly we find from the ${ }^{13} \mathrm{CO}(1-0)$ data a ratio $N\left(\mathrm{H}_{2}\right)$ LTE $/ W\left({ }^{13} \mathrm{CO}\right)$ of $5.4 \pm 0.210^{20} \mathrm{~cm}^{-2}\left(\mathrm{~K} \mathrm{~km} \mathrm{~s}^{-1}\right)^{-1}$. This number will be used to derive masses of clumps embedded in MBM 32.

The resulting masses in the three velocity intervals in Fig. 1a are $16.9 M_{\odot}\left(2-7 \mathrm{~km} \mathrm{~s}^{-1}\right), 4.1 M_{\odot}(-5-$ $\left.0 \mathrm{~km} \mathrm{~s}^{-1}\right)$, and $0.46 M_{\odot}\left(0-2 \mathrm{~km} \mathrm{~s}^{-1}\right)$, including a factor 1.36 for $\mathrm{He}$, and assuming a distance to the cloud of 100 pc. The cloud-averaged $\mathrm{H}_{2}$ densities for these three regions are then 70,78 , and $135 \mathrm{~cm}^{-3}$ respectively. It was assumed that the clouds are spherical, which is not very likely, and this may cause the lower mean density for the main cloud component, compared to the other two values. If the depth along the line of sight is smaller, the density for this component is larger than $56 \mathrm{~cm}^{-3}$. Maximum derived ${ }^{13} \mathrm{CO}$ column densities are about $1.510^{15} \mathrm{~cm}^{-2}$. MBM 32 has a low average extinction of $A_{B}=0.6 \mathrm{mag}$ over an area of $10^{\prime} 30^{\prime}$ (Heithausen \& Mebold 1989; see also Magnani \& de Vries 1986). The maximum value derived for the extinction (at a resolution of about $8^{\prime}$ ) is only slightly higher. Assuming a peak extinction $A_{V}$ of $0.8 \mathrm{mag}$ and using $N(\mathrm{H})=2.0610^{21} A_{V}$ (Bertoldi \& McKee 1992), the ${ }^{13} \mathrm{CO}$ abundance would be $1.810^{-6}$, equal to the Dickman value. However the assumption of a constant ${ }^{13} \mathrm{CO}$ abundance in a HLC such as MBM 32 is probably not correct. Van Dishoeck \& Black (1988) show that near $N\left(\mathrm{H}_{2}\right)=10^{21} \mathrm{~cm}^{-2}$ there is a strong increase of $\mathrm{CO}$ abundance with $N\left(\mathrm{H}_{2}\right)$. In addition part of the extinction can be related to $\mathrm{HI}$ gas rather than to $\mathrm{H}_{2}$ since the cloud is clearly detected in Hi (see Figs. 5-7). 

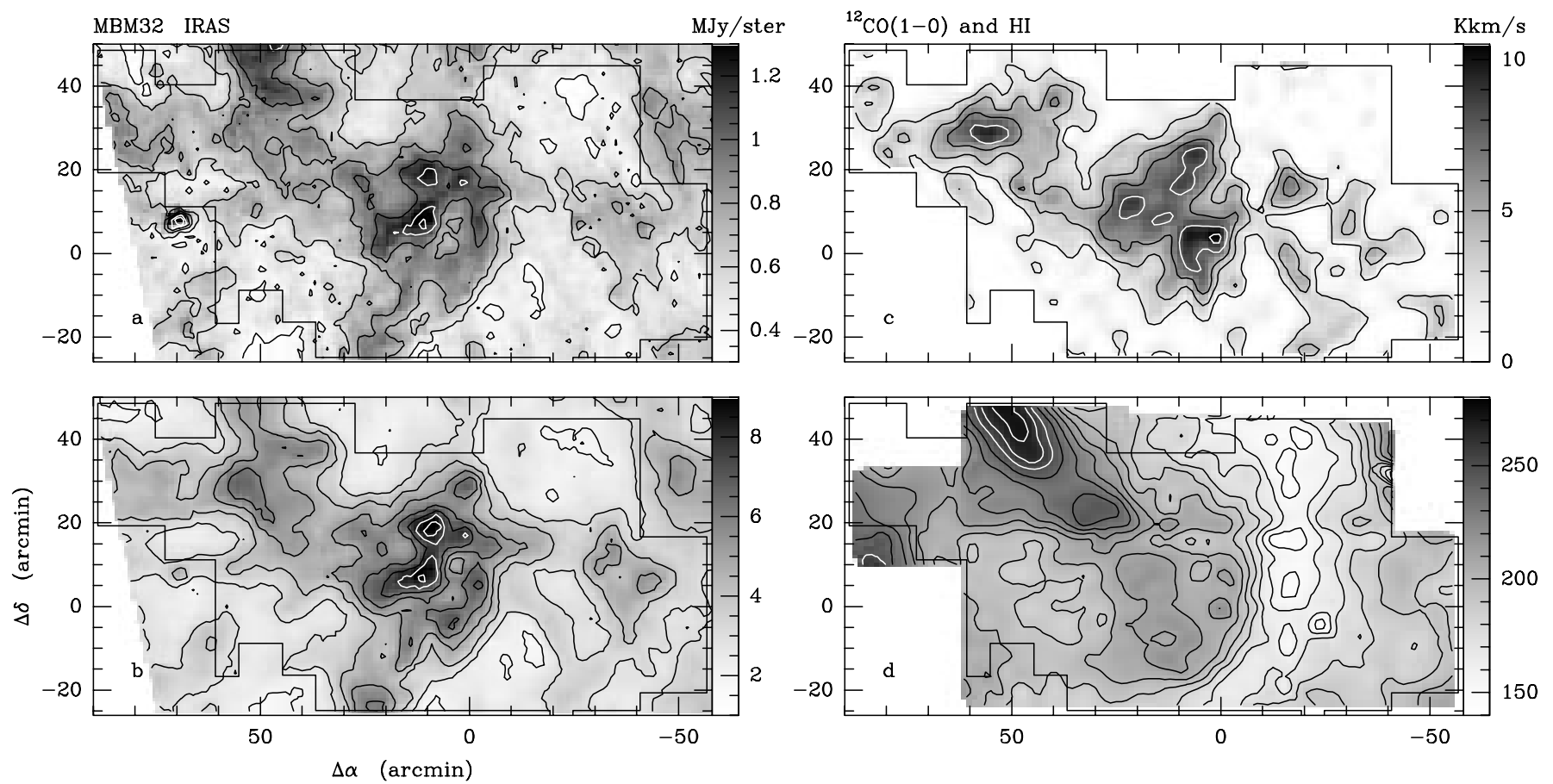

Fig. 9. a) Distribution of the IRAS $60 \mu \mathrm{m}$ emission associated with MBM 32. The contour levels are $0.4(0.2) 1.4 \mathrm{MJy}^{-1}$. b) The same for the $100 \mu \mathrm{m}$ emission. Contour levels are 2 (1) $9 \mathrm{MJy} \mathrm{sr}^{-1}$. The drawn lines indicate the area observed in ${ }^{12} \mathrm{CO}(1-0)$ with the MRS (Fig. 1a). c) Integrated ${ }^{12} \mathrm{CO}(1-0)$ emission over the velocity range -5 to $7 \mathrm{~km} \mathrm{~s}^{-1}$. Contour levels are $2(2) 10 \mathrm{~K} \mathrm{~km} \mathrm{~s}^{-1}$. d) Integrated Hi emission over the velocity range -30 to $+50 \mathrm{~km} \mathrm{~s}^{-1}$. Contour levels are 150 (10) $270 \mathrm{~K} \mathrm{~km} \mathrm{~s}^{-1}$

The derived $J=1-0$ excitation temperatures are between 5 and $9 \mathrm{~K}$, with the highest value of $9.6 \mathrm{~K}$ at offset $\left(4^{\prime}, 24^{\prime}\right)$. The kinetic temperature of $24 \mathrm{~K}$ derived by Schreiber et al. (1993) from $\mathrm{NH}_{3}$ observations therefore suggests that $\mathrm{CO}$ is subthermally excited. Also the dust temperature of $20 \mathrm{~K}$ is higher than $T_{\text {ex }}$. However also beam filling (the presence of many tiny clumps) could explain lower than expected line temparatures.

\subsection{Excitation}

To investigate the excitation of CO in MBM 32 we convolved the ${ }^{12} \mathrm{CO}(1-0)$ HRS and ${ }^{12} \mathrm{CO}(2-1)$ maps to the same angular and velocity resolution. We assumed that there is no emission outside the map area, so that the emission at the edge of the cloud would not be over estimated. From Gaussian fits to all spectra we derived the line ratios of the peak temperatures $(1-0) /(2-1)$ at all positions where both lines were detected. The results are shown in Fig. 12, where we distinguish between the three velocity components in the cloud $\left(2-7 \mathrm{~km} \mathrm{~s}^{-1}\right.$, $-5-0 \mathrm{~km} \mathrm{~s}^{-1}$, and $\left.0-2 \mathrm{~km} \mathrm{~s}^{-1}\right)$. For the main component $\left(2-7 \mathrm{~km} \mathrm{~s}^{-1}\right)$ the line ratios do not significantly vary with position in the cloud, but these ratios are somewhat lower than those of the other two components: the mean values of all positions are $1.93 \pm 0.41$ (s.d.; $N=301$ ) $\left(2-7 \mathrm{~km} \mathrm{~s}^{-1}\right), 2.40 \pm 0.81(N=60)\left(-5-0 \mathrm{~km} \mathrm{~s}^{-1}\right)$, and $2.72 \pm 0.80(N=12)\left(0-2 \mathrm{~km} \mathrm{~s}^{-1}\right)$.

${ }^{12} \mathrm{CO}(3-2)$ has been observed in a much smaller region due to the relative weakness of the lines. We compare it in the same way with the ${ }^{12} \mathrm{CO}(2-1)$ emission as above. However, because the $(3-2)$ transition has not been observed to the edge of the cloud, we did not add spectra without lines around the observed region before convolving to the lower resolution. This transition was only mapped in two small regions of the main cloud component $\left(2-7 \mathrm{~km} \mathrm{~s}^{-1}\right)$. The ratio $(2-1) /(3-2)$ of the peak $T_{\mathrm{R}}^{*}$ is $2.17 \pm 0.45(N=37)$ in the northern region, and $2.74 \pm 0.51(N=22)$ in the southern region. The difference in the two ratios might be real, but part of it might also be explained by the lower efficiency assumed for part of the measurements in the southern region (those made in 1990/1991). However for observations in this region we found no systematic differences in intensity after efficiency correction between observations made in 1990/1991 and in $1991 / 1992$. The ratio $(1-0) /(2-1)$ in both regions are equal within the uncertainty. As for this ratio (Fig. 14), the ratio $(2-1) /(3-2)$ is not dependent on the $T_{\mathrm{R}}^{*}(2-1)$ (see Sect. 4.3). 

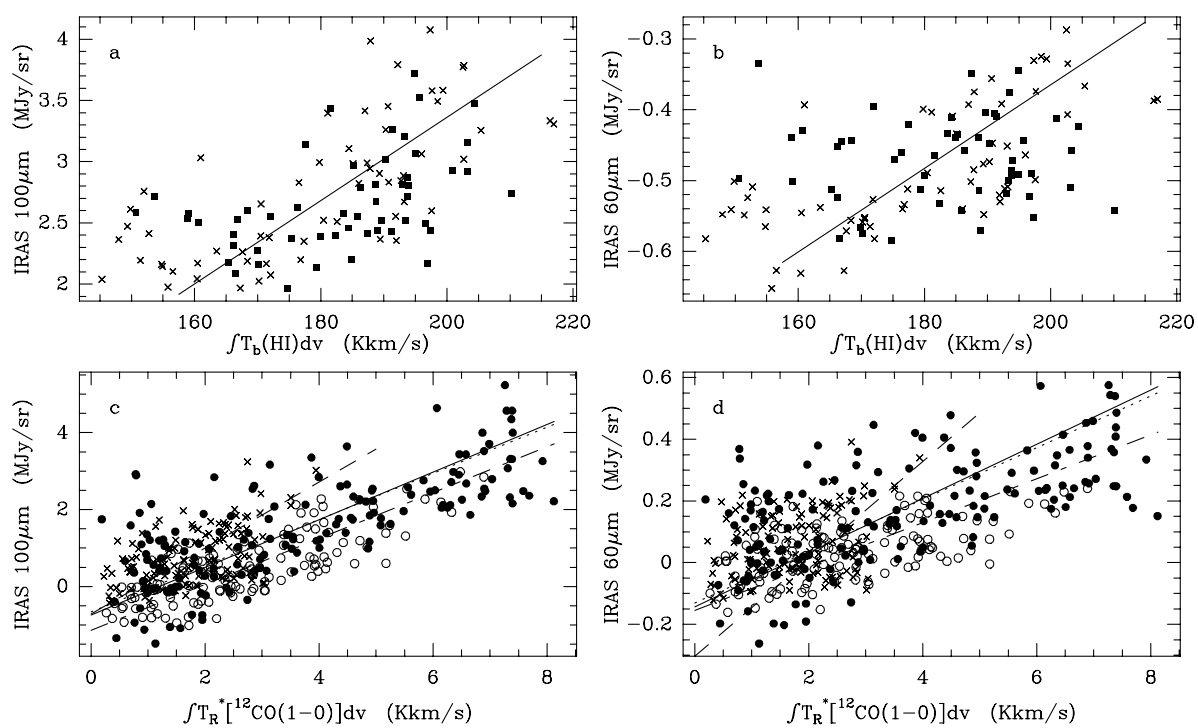

Fig. 10. a,b) Plot of IRAS (ISSA) $100 \mu \mathrm{m}$ a) and $60 \mu \mathrm{m} \mathrm{b}$ ) flux density versus integrated $\mathrm{HI}$ intensity of the local $\left(V_{\mathrm{lsr}}\right.$ near $\left.0 \mathrm{~km} \mathrm{~s}^{-1}\right)$ emission for lines of sight without ${ }^{12} \mathrm{CO}(1-0)$ emission. Crosses indicate positions with $\Delta \alpha<-10^{\prime}$. Filled squares indicate positions with $\Delta \alpha>-10^{\prime}$. The drawn line is the least squares fit through all data points. c,d) Plot of IRAS (ISSA) $100 \mu \mathrm{m} \mathrm{c}$ ) and $60 \mu \mathrm{m} \mathrm{d}$ ) flux density versus integrated ${ }^{12} \mathrm{CO}(1-0)$ intensity. The IRAS emission was corrected for Hi emission along each line of sight. The thick line is a least squares fit through all data points. The dashed line was obtained using positions with $\Delta \alpha<-10^{\prime}$ (crosses). The dotted line from positions with $-10^{\prime}<\Delta \alpha<34^{\prime}$ (filled circles), and the dash-dotted line from positions with $\Delta \alpha>34^{\prime}$ (open circles)

\subsection{A cut through $M B M 32$ in different transitions}

The ratio $(2-1) /(3-2)$ can be studied over a larger range in intensity at some positions where we have observed three transitions to a lower noise level than used for the maps. These are ${ }^{12} \mathrm{CO}(2-1),{ }^{13} \mathrm{CO}(2-1)$, and ${ }^{12} \mathrm{CO}(3-2)$. In these transitions we made a deep cut through part of the cloud at $\Delta \delta=0^{\prime}$. In ${ }^{12} \mathrm{CO}(2-1)$ the right ascension range was extended to cover also the negative velocity cloud. The results for both $(2-1)$ transitions are shown in Fig. 13, where the raster size is $1^{\prime}$. The upper two panels (Figs. 13a and b) show the ${ }^{12} \mathrm{CO}(2-1)$ emission of the negative and positive velocity components at the same scale. For the former one around $\alpha$-offset $-17^{\prime}$, the shapes of our spectra are consistent with similar deep ${ }^{12} \mathrm{CO}(1-0)$ spectra by Magnani et al. (1990) in this region: spectra at the west side of this cloud show additional velocity components at the blue side, and those at the east side have red wings. In the higher resolution $\left(30^{\prime \prime}\right){ }^{12} \mathrm{CO}(2-1)$ spectra of Magnani et al. (1990), these weak components are identified as separate small $(<0.03 \mathrm{pc})$ clumps. In Fig. 13b, also the weak negative velocity emission at positive offsets is visible. Figure $13 \mathrm{c}$ shows the ${ }^{13} \mathrm{CO}(2-1)$ emission in the same offset range as in Fig. 13b. Part of these data have a higher noise because of a shorter integration time.

The ratio of the ${ }^{12} \mathrm{CO}(2-1)$ and ${ }^{13} \mathrm{CO}(2-1)$ emission at the positions where the latter line was detected is shown in Fig. 14a. We show both the values determined from the peak intensities and those from the integrated intensity. It is seen that at the positions of strongest ${ }^{13} \mathrm{CO}$ emission at offsets of $5^{\prime}$ to $8^{\prime}$, the ratio is about 11 . At offsets west of this range the ration increases only slightly to values between 11 and 13 (with the exception of the position at offset $3^{\prime}$ ). However there is not a systematic increase in this range, as one might expect if the ${ }^{12} \mathrm{CO}$ optical depth decreases. This should be confirmed by deeper integrations in the most western part of the cloud. As in the case for the $(1-0)$ ratios, the ratios of the peak $T_{\mathrm{R}}^{*}$ are slightly higher (median 11.1) than those of the integrated $T_{\mathrm{R}}^{*}$ (median 14.2). Both ratios are higher than those of the $(1-0)$ ratios for the main cloud component (about 6 and 9 for peak and integrated $T_{\mathrm{R}}^{*}$, respectively; see Sect. 4.1) and higher than in GMCs (e.g. Kramer et al. 1996 found a ${ }^{13} \mathrm{CO} /{ }^{12} \mathrm{CO}(2-1)$ ratio of 1.5 to 8 for Orion $\left.\mathrm{B}\right)$. We can compare the $(2-1)$ ratios with those for the negative velocity component at offsets in the range $-1^{\prime}$ to $4^{\prime}$. Adding the emission in this range (omitting offset $3^{\prime}$ ) we find ratios of $19.4 \pm 4.3$ and $19.0 \pm 10.1$ respectively for the peak and integrated intensities of the negative velocity component (the peak ${ }^{13} \mathrm{CO}(2-1) T_{\mathrm{R}}^{*}$ is $0.028 \pm 0.015 \mathrm{~K}$ ). For the positive velocity component in this interval these values are $13.4 \pm 0.4$ and $13.3 \pm 1.1$. If the intrinsic ratio ${ }^{12} \mathrm{CO} /{ }^{13} \mathrm{CO}$ is equal to the typical value in the solar neighbourhood of $76 \pm 7$ (Wilson \& Rood 1994), the optical depths in both cloud components are very similar. Because of fractionation, the intrinsic ratio in the outer parts of a HLC such as MBM 32, will decrease slightly (see e.g. Fig. 6 of Turner et al. 1992), and possibly significantly (van Dishoeck et al. 1991 suggest a factor 2 to 3 ), while both the ${ }^{12} \mathrm{CO}$ and ${ }^{13} \mathrm{CO}$ abundance decrease to values much lower than the Dickman (1978) one (for $\left.{ }^{13} \mathrm{CO}\right)$. Deriving LTE column densities from the $(2-1)$ data along this line, we find values that are a factor $2-4$ lower than those from the $(1-0)$ HRS data at the same positions, while the derived excitation temperatures are almost equal for $(1-0)$ and $(2-1)$. This suggests that 


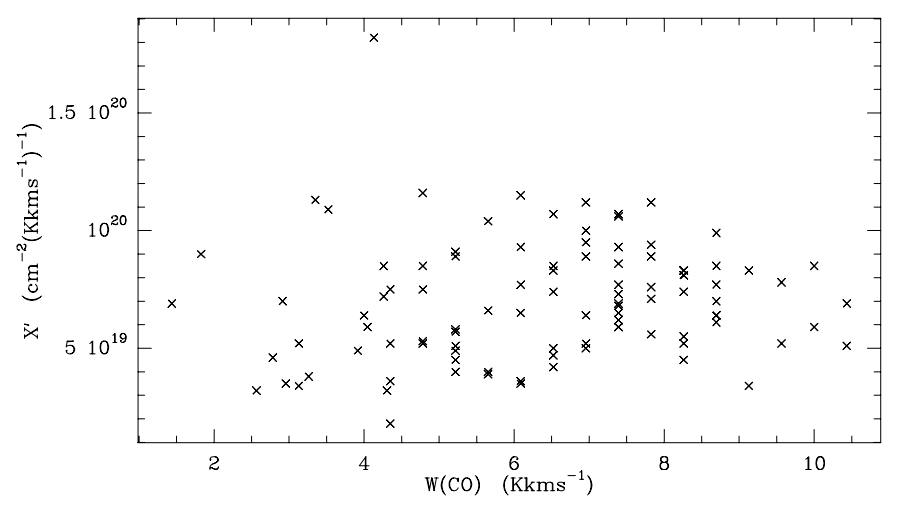

Fig. 11. Plot of factor $X^{\prime}=N\left(\mathrm{H}_{2}\right) / \int T \mathrm{~d} v\left({ }^{12} \mathrm{CO}(1-0)\right)$ versus $\int T \mathrm{~d} v\left({ }^{12} \mathrm{CO}(1-0)\right) \cdot X^{\prime}$ was derived from LTE calculations (using $J=1-0$ data) assuming a ${ }^{13} \mathrm{CO}$ abundance of $2.010^{-6}$

Table 2. Summary of line ratios and derived $\mathrm{X}$ ratios

\begin{tabular}{lrrr}
\hline & $V_{\text {lsr }}<0$ & $0<V_{\text {lsr }}<2$ & $V_{\text {lsr }}>2$ \\
\hline${ }^{12} \mathrm{CO}(1-0) /(2-1)$ & 2.40 & 2.72 & 1.93 \\
${ }^{12} \mathrm{CO}(2-1) /(3-2)$ & 2.1 & & 2.7 \\
${ }^{12} \mathrm{CO} /{ }^{13} \mathrm{CO}(1-0)\left(T_{\mathrm{R}}^{*}\right)$ & & & $5.2-6.9$ \\
${ }^{12} \mathrm{CO} /{ }^{13} \mathrm{CO}(1-0)\left(\int_{\mathrm{R}}^{*} \mathrm{~d} v\right)$ & & & $7-11$ \\
${ }^{12} \mathrm{CO} /{ }^{13} \mathrm{CO}(2-1)$ & 19.2 & 13.4 \\
$X^{\prime}\left(\right.$ lte) (for $\left.{ }^{12} \mathrm{CO}(1-0)\right)$ & $0.710^{20} a$ & \\
$X^{\prime}\left(\right.$ lte) (for $\left.{ }^{12} \mathrm{CO}(2-1)\right)$ & $1.410^{20} a$ & \\
$X^{\prime}($ lte $)\left(\right.$ for $\left.{ }^{13} \mathrm{CO}(1-0)\right)$ & $5.410^{20} a$ & \\
$X$ (fir) (for $\left.{ }^{12} \mathrm{CO}(1-0)\right)$ & $>0.210^{20} a$ & \\
& & \\
\hline
\end{tabular}

${ }^{a}$ Units $\mathrm{K} \mathrm{km} \mathrm{s}^{-1} \mathrm{~cm}^{-2}$.

the assumptions used for the LTE column densities are not correct, as was found also for other clouds, such as Orion (see e.g. Kramer et al. 1996). Since for deriving the used abundances similar assumptions were used, it has little influence on the derived cloud masses.

The distribution of the ${ }^{12} \mathrm{CO}(3-2)$ emission on a $40^{\prime \prime}$ raster along the same line as in Fig. 13 for $(2-1)$ is show in Fig. 15. The negative velocity emission (Fig. 15a) was observed with lower sensitivity than the positive velocity emission (Fig. 15b). An indication for a minimum near offset $+3^{\prime}$ in the latter panel is also seen in Fig. 13c. In all transitions the clouds edge appears not very sharp, but the decrease in intensity is steeper than exponential. We compare in Fig. 14b the ${ }^{12} \mathrm{CO}(2-1)$ and $(3-2)$ emission after convolution of the latter data to the $(2-1)$ angular resolution. Both cloud components show a line ratio which is independent of position. However the average values for both clouds are different: $2.77 \pm 0.23$ for the positive velocity emission at offsets larger than $-5^{\prime}$, and $2.09 \pm 0.21$ for the negative velocity emission. This difference is in the same direction as found for the two mapped regions (see Sect. 4.2). Both regions were again observed in different periods for which different telescope efficiencies were assumed.
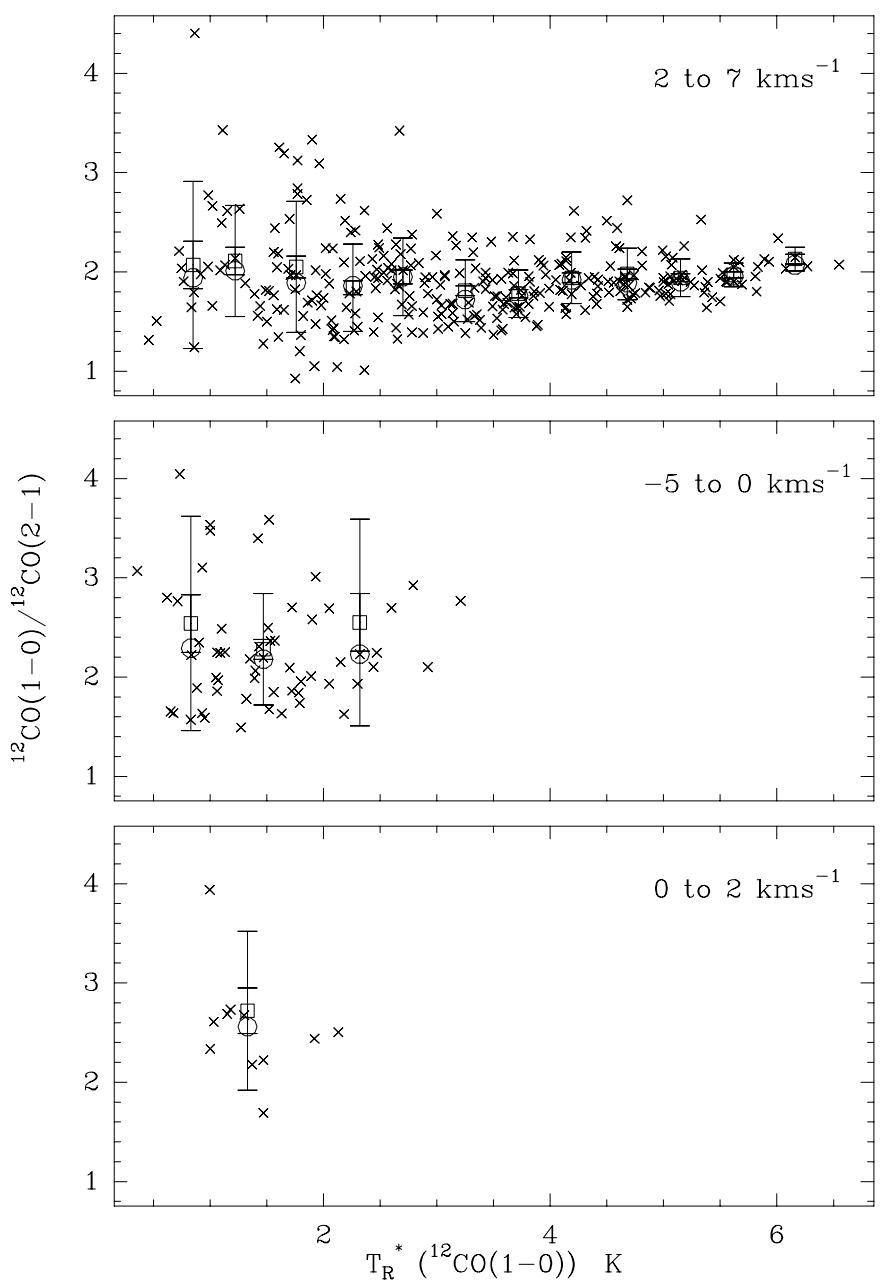

Fig. 12. The ratio of the peak $T_{\mathrm{R}}^{*}$ of ${ }^{12} \mathrm{CO}(1-0)$ and $(2-1)$ as a function of ${ }^{12} \mathrm{CO}(1-0)$ intensity for three velocity components in MBM 32. Indicated are average (squares) and median (circles) values for different intervals of $T_{\mathrm{R}}^{*}$, together with the standard deviations and mean errors

In Table 2 we summarize the measured average line ratios in the different parts of the cloud as well as working values for $X^{\prime}$ for the different transitions which result in column densities equal to the LTE column densities (for ${ }^{12} \mathrm{CO}(2-1)$ we assumed a $(1-0) /(2-1)$ ratio of 2 to obtain this $X^{\prime}$ ). The ratios can be compared with average $\int T \mathrm{~d} v$ ratios listed by Ingalls et al. (2000) for translucent clouds (obtained from data by van Dishoeck et al. (1991): $(1-0) /(2-1)=1.30_{-0.29}^{+0.59},(2-1) /(3-2)=1.82_{-0.41}^{+0.74}$. Their ${ }^{12} \mathrm{CO} /{ }^{13} \mathrm{CO}(1-0)$ ratio is $7.7_{-1.8}^{+3.4}$. Our ratios appear to be larger, but might be consistent if calibration uncertainties are taken into account.

We have tried to analyze these ratios with an escape probability model (Stutzki \& Winnewisser 1985). It appears that the ${ }^{12} \mathrm{CO}$ line ratios are consistent with low density $\left(n<100 \mathrm{~cm}^{-3}\right)$ gas with $T_{\text {kin }}$ about $12 \mathrm{~K}$. Also the strongest ${ }^{12} \mathrm{CO}(1-0)$ lines of about $6 \mathrm{~K}$ can be explained by such gas. However the weaker ${ }^{12} \mathrm{CO}$ lines with the same line ratios cannot be explained. It is possible that 

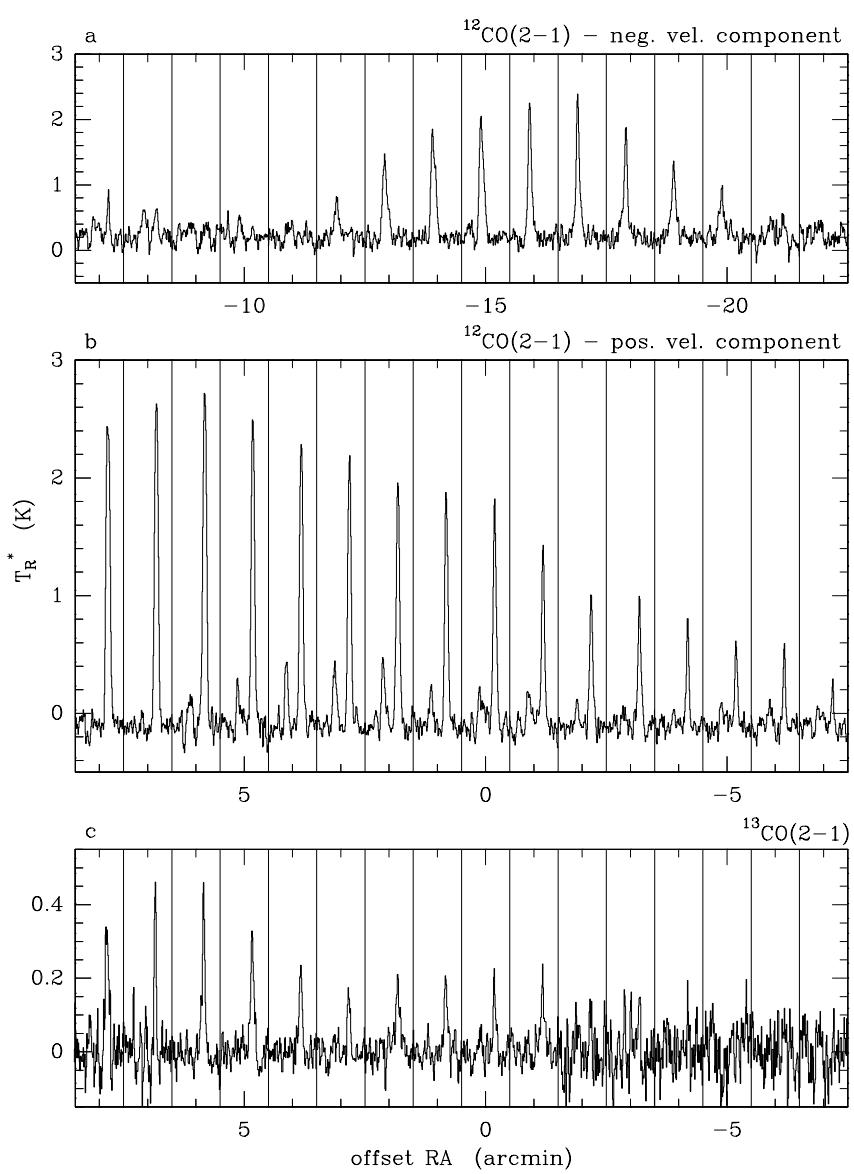

Fig. 13. a) ${ }^{12} \mathrm{CO}(2-1)$ emission distribution along a cut in right ascension at $\Delta \delta=0^{\prime}$ (with respect to $\alpha(1950)=9^{\mathrm{h}} 28^{\mathrm{m}} 42^{\mathrm{s}}$, $\left.\delta(1950)=+66^{\circ} 5^{\prime}\right)$ for the negative velocity component, and b) for the positive velocity component. The range in $V_{\mathrm{lsr}}$ is -10 to $10 \mathrm{~km} \mathrm{~s}^{-1}$ for each spectrum. c) The same as b), but for ${ }^{13} \mathrm{CO}(2-1)$

this is caused by similar gas with a smaller beam filling factor. But then the relatively high ${ }^{12} \mathrm{CO} /{ }^{13} \mathrm{CO}(1-0)$ ratios still cannot be explained. The results of the analysis of the line intensities towards one central position in MBM 32 by Schreiber et al. (1993), which indicate a flat density distribution and the constancy of the line ratios within the whole cloud, may suggest that the cloud consist of small clumps (much smaller than the beam size) with average properties that do not change within the mapped area. However the number of such clumps decreases towards the cloud edges. The presence of small clumps within a beam was also suggested by Tauber et al. (1991) from high spectral resolution ${ }^{12} \mathrm{CO}$ and ${ }^{13} \mathrm{CO}(1-0)$ measurements towards Orion. Ingalls et al. (2000) conclude from the constant line rations in translucent clouds (including their own ${ }^{12} \mathrm{CO}(4-3)$ data) that the clouds consist of high density $\left(n\left(\mathrm{H}_{2}\right)=10^{4.5} \mathrm{~cm}^{-3}\right)$, low temperature $(8 \mathrm{~K})$ cells. However we note that they only used the observed line ratios to compare with their LVG models, no line tem-
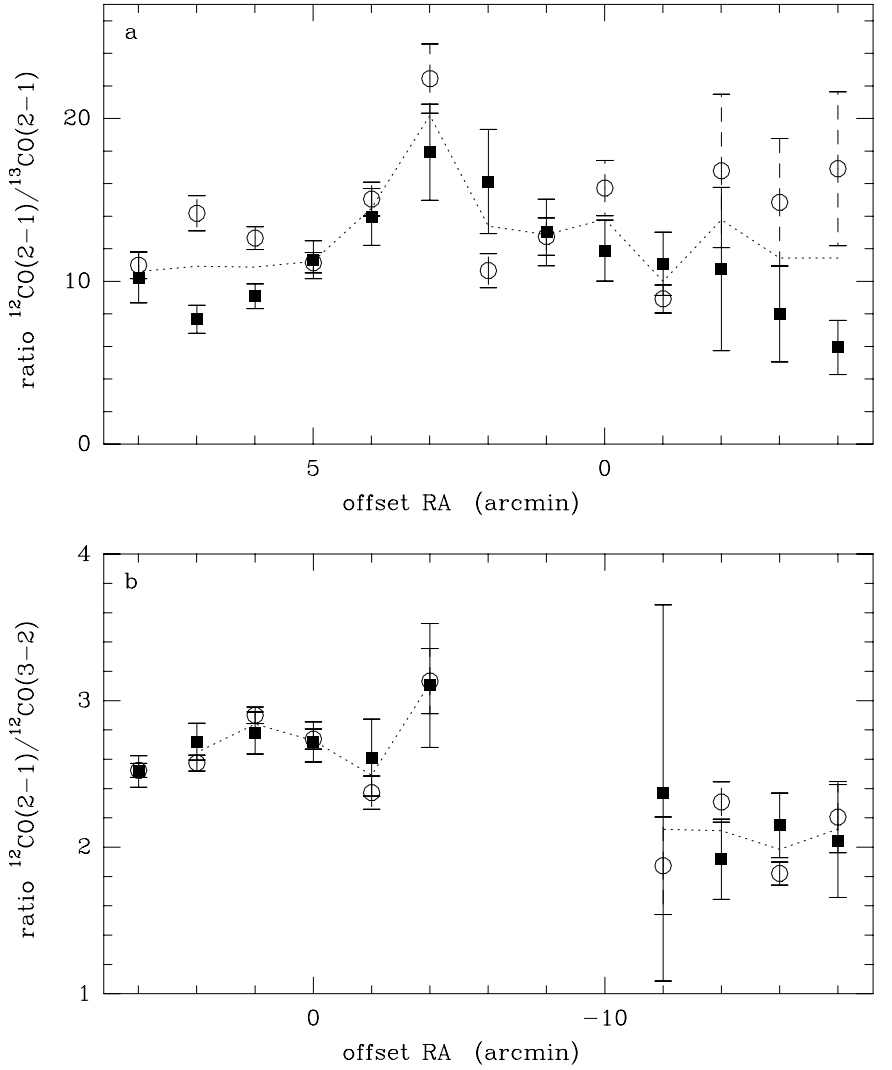

Fig. 14. a) The line ratios ${ }^{12} \mathrm{CO}(2-1) /{ }^{13} \mathrm{CO}(2-1)$ along the cut through MBM 32 in Fig. 14b. The filled squares indicate the ratios for the peak $T_{\mathrm{R}}^{*}$, and the open circles for $\int T_{\mathrm{R}}^{*}$ $\mathrm{d} v$. The dashed line connects the averages of the two values. b) The same for the ratio ${ }^{12} \mathrm{CO}(2-1) /{ }^{12} \mathrm{CO}(3-2)$

peratures. We found that in that case solutions are easier to find (see above).

\subsection{Cloud structure}

We have analysed the $\mathrm{CO}$ datacubes with the Gaussclumps algorithm developed by Stutzki \& Güsten (1990). A detailed discussion of this algorithm and its results for different clouds is given by Kramer et al. (1998). The steering parameters were varied in the range found by these authors for their clouds, and those values were taken which resulted in clumps having realistic sizes and linewidths. However the fit-results are not unique - the derived clump parameters depend slightly on the steering parameters. This analysis was done for our ${ }^{12} \mathrm{CO}(1-0$; MRS and HRS $),{ }^{13} \mathrm{CO}(1-0)$, and ${ }^{12} \mathrm{CO}(2-1)$ cubes. Before analysing it, the ${ }^{13} \mathrm{CO}(1-0)$ data cube was filled by interpolation to a fully sampled $2^{\prime}$ cube. The results are given in Table 3 for ${ }^{12} \mathrm{CO}(1-0$, MRS $)$, Table 4 for ${ }^{12} \mathrm{CO}(1-0$, HRS $)$, Table 5 for ${ }^{12} \mathrm{CO}(2-1$, MRS $)$ and Table 6 for ${ }^{13} \mathrm{CO}(1-0$, HRS $)$. The limits for linewidths and $T_{\mathrm{R}}^{*}$ mentioned in the table header are uncorrected for resolution. Listed are position (offset with respect 
Table 7. Summary of the results

\begin{tabular}{lrrr}
\hline & $V_{\text {lsr }}<0$ & $0<V_{\text {lsr }}<2$ & $V_{\text {lsr }}>2$ \\
\hline$T_{\mathrm{R}}^{*}\left({ }^{12} \mathrm{CO}(1-0)_{\text {peak }}\right)$ & & & \\
$\delta v(\mathrm{CO})$ & $2.6 \mathrm{~K}$ & $1.3 \mathrm{~K}$ & $4.8 \mathrm{~K}$ \\
$\delta v(\mathrm{HI})$ & $1.92 \mathrm{~km} \mathrm{~s}^{-1}$ & $2.1 \mathrm{~km} \mathrm{~s}^{-1}$ & $1.12-1.49 \mathrm{~km} \mathrm{~s}^{-1}$ \\
$r_{\text {eq }}(\mathrm{CO})$ & $4.35 \mathrm{~km} \mathrm{~s}^{-1}$ & $4.75 \mathrm{~km} \mathrm{~s}^{-1}$ & $4.95 \mathrm{~km} \mathrm{~s}^{-1}$ \\
$M_{\text {gas }}($ from CO; no He) & $0.57 \mathrm{pc}$ & $0.23 \mathrm{pc}$ & $0.95 \mathrm{pc}$ \\
$M_{\text {gas }}$ (from Hi; no He) & $3.04 M_{\odot}$ & $0.34 M_{\odot}$ & $12.4 M_{\odot}$ \\
$M_{\text {dust }}$ & $2.61 M_{\odot}$ & $1.23 M_{\odot}$ & $8.38 M_{\odot}$ \\
& $0.020 M_{\odot}$ & & $0.053 M_{\odot}$ \\
\hline
\end{tabular}
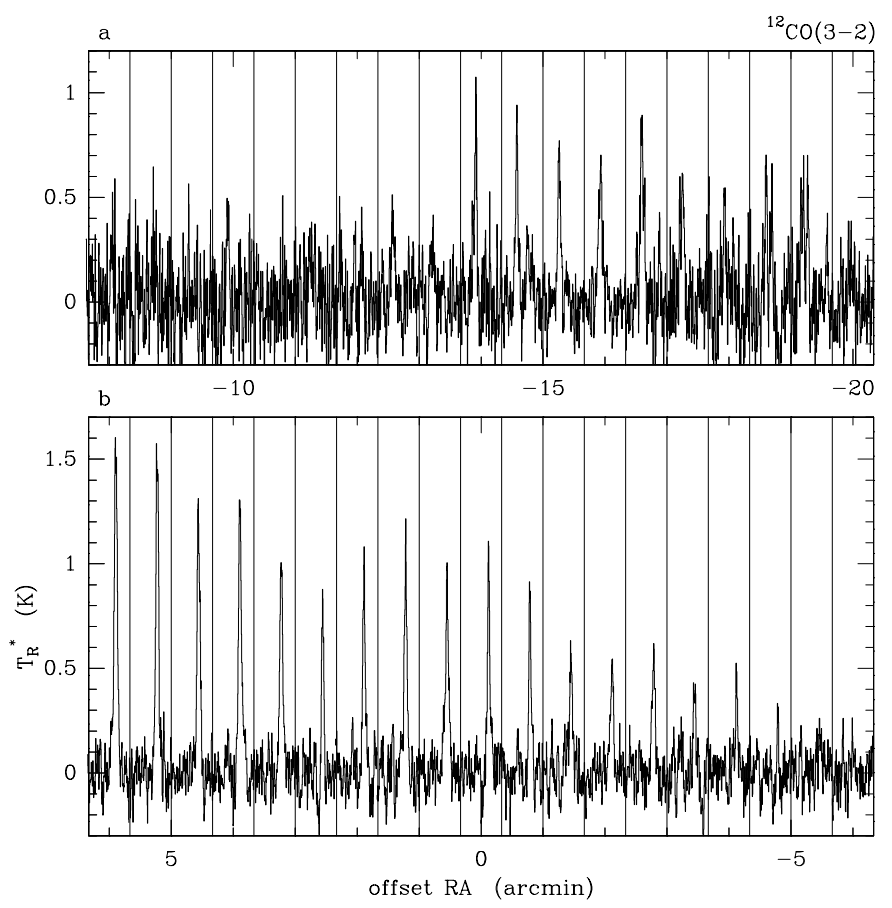

Fig. 15. ${ }^{12} \mathrm{CO}(3-2)$ emission distribution along a cut in right ascension at $\Delta \delta=0^{\prime}$ with respect to $\alpha(1950)=9^{\mathrm{h}} 28^{\mathrm{m}} 42^{\mathrm{s}}$, $\delta(1950)=+66^{\circ} 5^{\prime}$ for the negative velocity component $\mathbf{a}$ ), and for the positive velocity component $\mathbf{b})$. The range in $V_{\mathrm{lsr}}$ is -10 to $10 \mathrm{~km} \mathrm{~s}^{-1}$ for each spectrum

to $\left.\alpha(1950)=9^{\mathrm{h}} 28^{\mathrm{m}} 42^{\mathrm{s}}, \delta(1950)=+66^{\circ} 5^{\prime}\right)$, peak $T_{\mathrm{R}}^{*}$, Gaussian half width size, velocity, linewidth, $W_{\mathrm{CO}}$ mass [using appropriate " $X$ " factors of $0.710^{20}\left({ }^{12} \mathrm{CO}(1-0)\right)$, $1.410^{20}\left({ }^{12} \mathrm{CO}(2-1)\right)$, and $5.410^{20} \mathrm{~cm}^{-2}\left(\mathrm{~K} \mathrm{~km} \mathrm{~s}^{-1}\right)^{-1}$ $\left.\left({ }^{13} \mathrm{CO}(1-0)\right)\right]$, average density, and virial mass. Based on the results of Schreiber et al. (1993) we used for the virial masses the assumption of a constant density cloud: $M_{\mathrm{vir}}=210 \delta v^{2} r$. Both the average radius $r$, and the line width $\delta v$ were corrected for the angular and spectral resolution, respectively. The peak $T_{\mathrm{R}}^{*}$ was corrected for both effects. We indicated ( + in Col. 10) the clumps that are clearly visible in the original channel maps (without other clumps being subtracted first). These are in general only the strongest ones. We distinguish again the three velocity ranges (see e.g. Fig. 1a), and we only list clumps stronger than the $3 \sigma$ level. They should have line widths significantly above the channel width and be larger than the beam size at least in one direction. Ideally, the clumps listed in Tables 3 to 6 should be the same. We find that the clumps are found in the same general area, but the details differ due to e.g. differences in resolution, and to noise and pointing errors. Comparing the total mass of the clumps found in this way with the total cloud mass in the relevant velocity range it is seen that $40-50 \%$ of the cloud is in such clumps. The rest of the mass is in smaller clumps (or clumps that are not Gaussian in shape). This fraction is smaller for the weaker cloud components (such as the one in the range $0-2 \mathrm{~km} \mathrm{~s}^{-1}$ ). The largest clumps have a size of $0.5 \mathrm{pc}$ and a mass of about $2 M_{\odot}$. Mean $\mathrm{H}_{2}$ densities in the clumps are several $100 \mathrm{~cm}^{-3}$ and higher. It is seen that for all clumps the virial mass is much larger than the mass derived using " $X$ ". Whereas it is possible that this means that these clumps are not bound, Turner et al. (1989) argue that this difference becomes much less if one takes external pressure on the clumps due to interclump gas into account.

Pound \& Blitz (1993) list 3 clumps found in a still unpublished ${ }^{13} \mathrm{CO}(1-0)$ map of MBM 32. Two of those clumps are located close to clumps listed in Table 6. Our radii are larger because of the angular resolution of the observations.

We have also tried to analyse the cloud data with more detailed algorithms, investigating for instance the power law distribution of clump masses (see e.g. Stutzki et al. 1998). However the data cubes are not large enough to allow us to obtain reliable results.

Similar to the CO maps, we have investigated the structure of the Hi gas. We used a data cube where the broad velocity component had been subtracted. The algorithm finds about 60 clumps larger than the 9 '.2 resolution. The residual map shows fairly uniform emission with lines of several K. The largest clump found represents the $24 \mathrm{~K}$ emission at $3.8 \mathrm{~km} \mathrm{~s}^{-1}$ associated with the main part of the molecular cloud. However, most clumps are located at the edge of the mapped region and therefore the data are of no use for a detailed analysis.

In Fig. 16 we compare some of the derived parameters (size, linewidth and mass) of the clumps listed in Tables 3 to 6 . We distinguish between the three velocity components and between the clumps which are visible in 

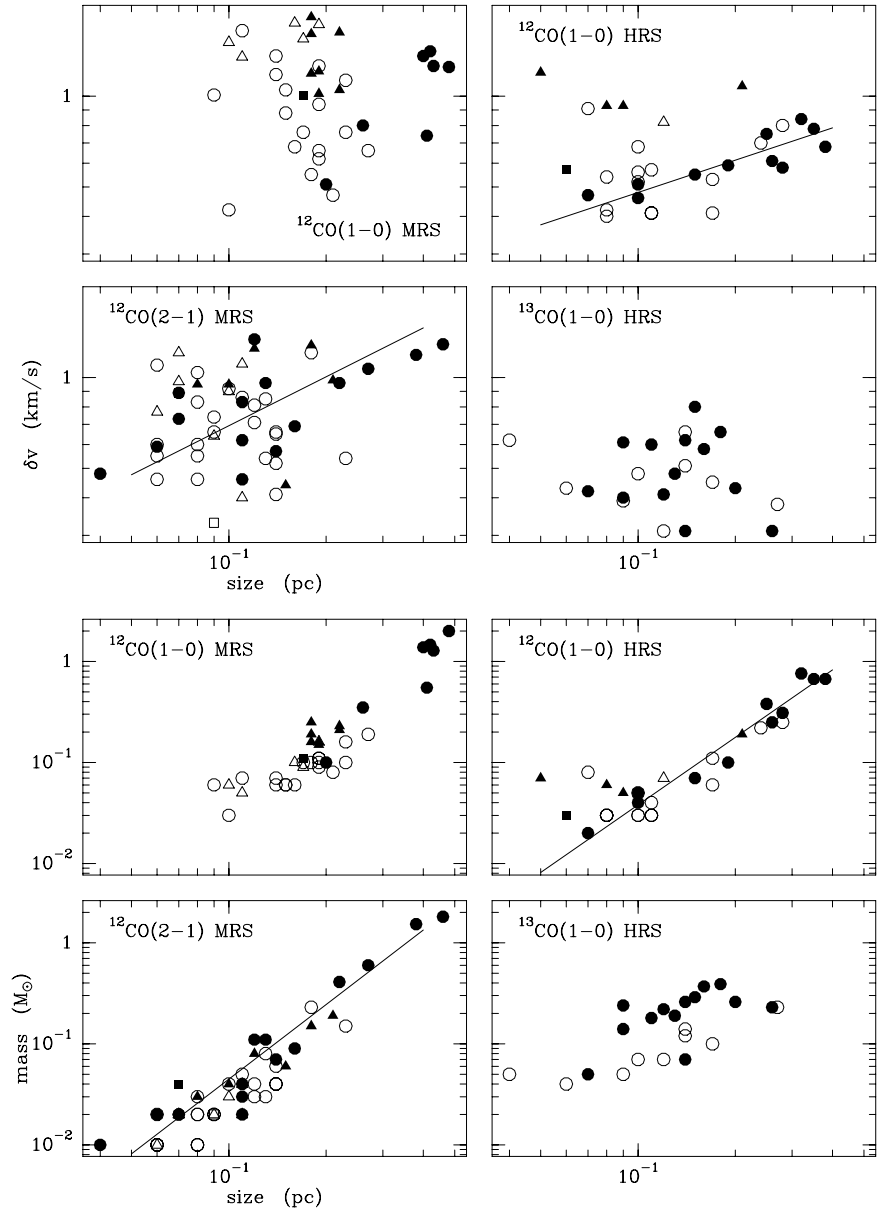

Fig. 16. a) Correlation of clump size and line width from the analysis of ${ }^{12} \mathrm{CO}(1-0)$ MRS, ${ }^{12} \mathrm{CO}(1-0) \mathrm{HRS},{ }^{12} \mathrm{CO}(2-1)$ MRS, and ${ }^{13} \mathrm{CO}(1-0)$ HRS data. The circles indicate clumps found in the main cloud component, squares are clumps in the small positive velocity component, and triangles are clumps in the negative velocity component. Filled symbols are clumps seen in the original maps (+ in Col. 10 of Tables 3 to 6 ). b) The same for the correlation between clump size and clump mass. The drawn lines indicate the fits to the filled circles (Eqs. (4) to $(7))$

the original maps before subtracting other Gaussian clumps and those which are not visible in those maps. It is seen in Fig. 16a that the ${ }^{12} \mathrm{CO}(1-0)$ linewidths found from the MRS data are somewhat larger than those derived from the HRS data, even after corection for the resolution. There is no significant difference in distribution between the filled and open symbols $(+$ and - , respectively, in Col. 10 of Tables 3 to 6). We can investigate the linear correlation between the parameters for those velocity components and transitions where the number of clumps found is large enough. We only use the clumps indicated by the filled symbols. These results can be compared with correlations found for integral parameters of HLC. For the main component $\left({ }^{12} \mathrm{CO}(1-0)\right.$
HRS data) we obtained (correlation coefficient 0.86):

$$
\log (\delta v)=(0.04 \pm 0.04)+(0.36 \pm 0.05) \log (\text { size }) .
$$

The ${ }^{12} \mathrm{CO}(2-1)$ data for the main component gave (correlation coefficient 0.67$)$ :

$$
\log (\delta v)=(0.38 \pm 0.09)+(0.54 \pm 0.10) \log (\text { size }) .
$$

The range in size or the number of data points is too small for the other transitions and components. The average slope of Eqs. (4) and (5), is within the uncertainties equal to the slope found for individual HLC's $(0.5 \pm 0.2)$ or for a sample of known clouds including also GMC's $(0.46 \pm 0.03)$ (see Heithausen 1996). This could suggest that the density distribution within a clump is the same as the average density distribution within a cloud (if there are no pressure gradients; see Heithausen 1996).

The data points in Fig. 16b show a larger correlation which is visible in all four panels. We obtain from the same two data sets as above the following results:

$$
\log (\operatorname{mass})=(0.80 \pm 0.09)+(2.22 \pm 0.11) \log (\text { size })
$$

$\left({ }^{12} \mathrm{CO}(1-0) \mathrm{HRS}\right.$ data; correlation coefficient 0.98).

$$
\log (\operatorname{mass})=(1.10 \pm 0.12)+(2.45 \pm 0.16) \log (\text { size })
$$

$\left({ }^{12} \mathrm{CO}(2-1)\right.$ MRS data; correlation coefficient 0.96$)$.

The mean slope in Eqs. (6) and (7) of about +2.3 is somewhat steeper than the one found for GMC's of 2.0 by Brand \& Wouterloot (1995) although with a much smaller constant term, which reflects the lower densities of the clumps. The slope is equal to the one found by Heithausen et al. (1998b) towards the Polaris Flare from the combination of different data sets.

\section{Conclusions}

We have mapped (parts of) the HLC MBM 32 in ${ }^{12} \mathrm{CO}(1-0),(2-1)$, and $(3-2)$, and in ${ }^{13} \mathrm{CO}(1-0)$ and $(2-1)$. A summary of our results is given in Table 7 .

Our ${ }^{12} \mathrm{CO}(1-0)$ map of MBM 32 shows that the cloud consists of three components, which can be distinguished by their radial velocity. The main (eastern) component, at $V_{\mathrm{lsr}}>2 \mathrm{~km} \mathrm{~s}^{-1}$ has a mass (derived from ${ }^{12} \mathrm{CO}(1-0)$ data) of about $16.9 M_{\odot}$. The (western) component at $V_{\text {lsr }}<0 \mathrm{~km} \mathrm{~s}^{-1}$ has $4.1 M_{\odot}$, and the emission in the range in between both velocities has $0.46 M_{\odot}$.

The CO spectra are fairly simple and contain no signs of self-absorption or strong, broad line wings. At most positions only one velocity component is detected, except for the main component which is overlapping with the eastern component. Within the main component there is possibly some overlap between emission at $2.0 \mathrm{~km} \mathrm{~s}^{-1}$ and at $3.5 \mathrm{~km} \mathrm{~s}^{-1}$.

Strong Hi emission is found from 100-m Effelsberg measurements towards the eastern component, but spectra show that there is Hi emission associated with all three 
molecular components. The mass of Hi gas is about equal to that of the $\mathrm{H}_{2}$. For some of the emission we see velocity differences between $\mathrm{CO}$ and $\mathrm{Hi}$ emission between -1.5 and $+1.5 \mathrm{~km} \mathrm{~s}^{-1}$.

Correcting the FIR (60 and $100 \mu \mathrm{m}$ ) emission for contribution of dust associated with the Hi there is a good correlation between dust and $\mathrm{CO}$ emission. The $X$ ratio $N\left(\mathrm{H}_{2}\right) / \int T_{\mathrm{R}}^{*}\left({ }^{12} \mathrm{CO}(1-0)\right) \mathrm{d} v$ derived from the combination of CO, FIR, and Hi data $\left(0.210^{20}\right)$ is lower than that derived assuming LTE from the CO data alone $\left(0.710^{20}\right)$. Part of the differences might be due to the adopted ${ }^{13} \mathrm{CO}$ abundance, which is uncertain in HLCs.

From the ratio of 60 and $100 \mu \mathrm{m}$ emission we derive a constant dust temperature of $20 \mathrm{~K}$. The ratio of gas to dust mass, 236, is lower than derived from FIR-CO comparisons in other clouds. This suggests that there is relatively less cold dust.

Apart from the ratio ${ }^{12} \mathrm{CO}(1-0) /{ }^{13} \mathrm{CO}(1-0)$, line ratios of different $\mathrm{CO}$ transitions appear to be constant within different cloud components. In particular this is found from deep measurements along a cut through the cloud in ${ }^{12} \mathrm{CO}(2-1)$ and $(3-2)$, and in ${ }^{13} \mathrm{CO}(2-1)$. The ratio ${ }^{12} \mathrm{CO} /{ }^{13} \mathrm{CO}$ of $T_{\mathrm{R}}^{*}$ and of $\int T_{\mathrm{R}} R^{*} \mathrm{~d} v$ is higher than typical ratios in galactic giant molecular clouds, indicating the lower optical depth of $\mathrm{CO}$. The constant line ratios may suggest a cloud model consisting of small $(<<$ beam size) clumps, with approximately the constant size and density distribution within the cloud, where the line intensities are determined by the clump filling factors.

The emission was subdivided in Gaussian clumps. For the main components typically $40-50 \%$ of the mass is found to be in clumps with $T_{\mathrm{R}}^{*}$ above a $3 \mathrm{rms}$ level. The size of the clumps is between about $0.4 \mathrm{pc}$ and the resolution limit of $\approx 0.07 \mathrm{pc}$. From the ${ }^{12} \mathrm{CO}(1-0)$ and $(2-1)$ data we obtain for these clumps a correlation $\delta v \propto r^{0.45}$, close to values found for other cloud samples.

Acknowledgements. We thank all KOSMA-observers who observed MBM 32 when their own sources were below the horizon. Comments on an earlier version of this paper by J. Brand and C. Kramer are appreciated.

The KOSMA radio telescope at Gornergrat-Süd observatory is operated by the University of Köln, and supported by the Deutsche Forschungsgemeinschaft through grant SFB-301, as well as by special funding from the Land Nordrhein-Westfalen. The observatory is administered by the Internationale Stiftung Hochalpine Forschungsstationen Jungfraujoch und Gornergrat, Bern, Switzerland.

\section{References}

Bertoldi F., McKee C.F., 1992, ApJ 395, 140

Brand J., Wouterloot J.G.A., 1995, A\&A 303, 851

Brand J., Wouterloot J.G.A., 1998, A\&A 337, 539 de Vries H.W., Heithausen A., Thaddeus P., 1987, ApJ 319, 723

Dickman R.L., 1978, ApJS 37, 407

Digel S.W., Grenier I.A., Heithausen A., Hunter S.D., Thaddeus P., 1996, ApJ 463, 609

Heithausen A., 1996, A\&A 314, 251

Heithausen A., Mebold U., 1989, A\&A 214, 347

Heithausen A., Mebold U., de Vries H.W., 1987, A\&A 179, 263

Heithausen A., Corneliussen U., Großmann V., 1998a, A\&A 330,311

Heithausen A., Bensch F., Stutzki J., Falgarone E., Panis J.F., 1998b, A\&A 331, L65

Herbstmeier U., Heithausen A., Mebold U., 1993, A\&A 272, 514

Hunter S.D., Bertsch D.L., Catelli J.R., et al., 1997, ApJ 481, 205

Ingalls J.G., Bania T.M., Lane A.P., Rumitz M., Stark A.A., 2000, ApJ (in press)

Isobe T., Feigelson E.D., Akritas M.G., Babu G.J., 1990, ApJ 364, 104

Kalberla P.M.W., Mebold U., Reif K., 1982, A\&A 106, 190

Kramer C., Stutzki J., Winnewisser G., 1996, A\&A 307, 915

Kramer C., Stutzki J., Röhrig R., Corneliussen U., 1998, A\&A 329,249

Kun M., 1992, A\&AS 92, 875

Magnani L., de Vries C.P., 1986, A\&A 168, 271

Magnani L., Onello J.S., 1995, ApJ 443, 169

Magnani L., Blitz L., Mundy L., 1985, ApJ 295, 402

Magnani L., Carpenter J.M., Blitz L., Kassim N.E., Nath B.B., 1990, ApJS 73, 747

Mebold U., Heithausen A., Reif K., 1987, A\&A 180, 213

Meyerdierks H., Heithausen A., 1996, A\&A 313, 929

Pound M.W., Blitz L., 1993, ApJ 418, 328

Schreiber W., Wouterloot J.G.A., Heithausen A., Winnewisser G., 1993, A\&A 276, L5

Strong A.W., Mattox J.R., 1996, A\&A 308, L21

Stutzki J., Güsten R., 1990, ApJ 356, 513

Stutzki J., Winnewisser G., 1985, A\&A 144, 13

Stutzki J., Bensch F., Heithausen A., Ossenkopf V., Zielinsky M., 1998, A\&A 336, 697

Tauber J., Goldsmith P.F., Dickman R.L., 1991, ApJ 375, 635

Turner B.E., Rickard L.J., Xu L.P., 1989, ApJ 344, 292

Turner B.E., Xu L.P., Rickard L.J., 1992, ApJ 391, 158

van Dishoeck E.F., Black J.H., 1988, ApJ 334, 771

van Dishoeck E.F., Black J.H., Philips T.G., Gredel R., 1991, ApJ 366, 141

Verter F., Rickard L.J., 1998, AJ 115, 745

Vladilo G., Centurión M., de Boer K.S., et al., 1994, A\&A 291, 425

Williams D.R., 1973, A\&AS 8, 505

Wilson T.L., Rood R.T., 1994, ARA\&A 32, 191

Winnewisser G., Bester M., Ewald R., et al., 1986, A\&A 167, 207

Winnewisser G., Zimmermann P., Hernichel J., et al., 1990, A\&A 230, 248

Wood D.O.S., Myers P.C., Daugherty D.A., 1994, ApJS 95, 457 